\title{
Gradhiva
}

GRADHIV

Revue d'anthropologie et d'histoire des arts

$31 \mid 2020$

L'idéal du musicien et l'âpreté du monde

\section{Quand l'art fait loi : histoires juridiques de Piotr Pavlenskiy en Russie et en France}

When Art Has the Force of Law: legal stories by Piotr Pavlenskiy in Russia and France

\section{Nataliya Tchermalykh}

\section{(2) OpenEdition}

Journals

Édition électronique

URL : http://journals.openedition.org/gradhiva/5117

DOI : $10.4000 /$ gradhiva. 5117

ISSN : $1760-849 x$

Éditeur

Musée du quai Branly Jacques Chirac

Édition imprimée

Date de publication : 2 septembre 2020

Pagination : 145-165

ISBN : 978-2-35744-131-6

ISSN : 0764-8928

\section{Référence électronique}

Nataliya Tchermalykh, «Quand l'art fait loi : histoires juridiques de Piotr Pavlenskiy en Russie et en France », Gradhiva [En ligne], 31 | 2020, mis en ligne le 31 mars 2021, consulté le 02 avril 2021. URL http://journals.openedition.org/gradhiva/5117; DOI : https://doi.org/10.4000/gradhiva.5117 


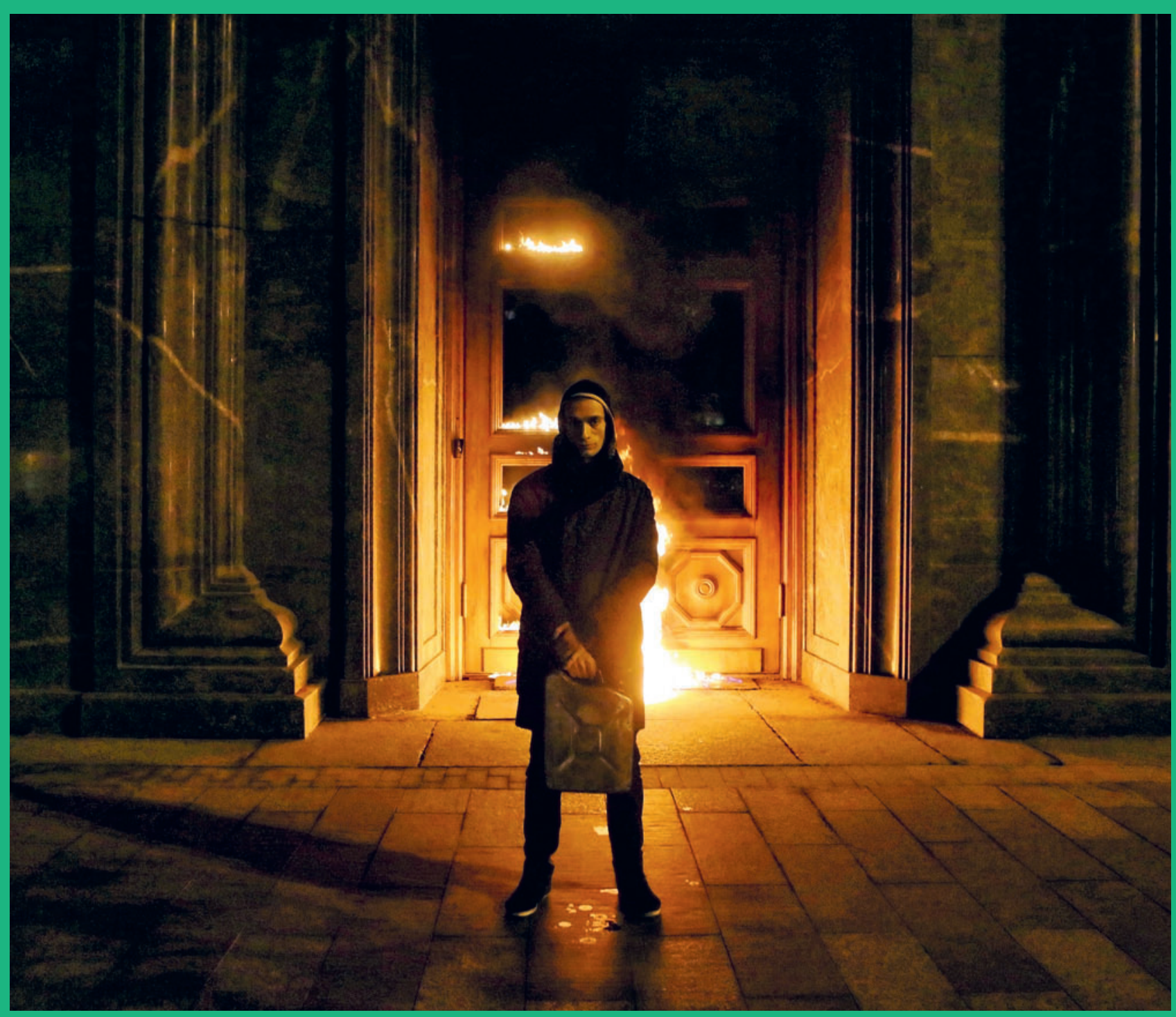


Études et essais

Quand l'art fait loi : histoires juridiques de Piotr Pavlenskiy en Russie et en France

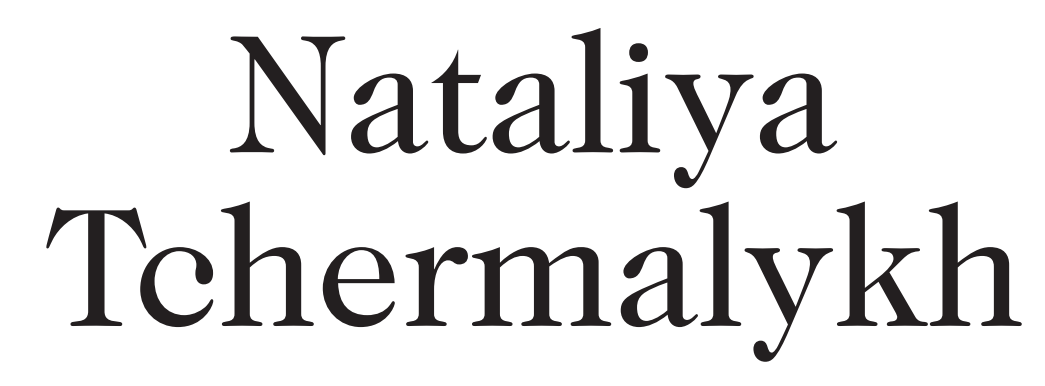

Nataliya

Tchermalykh 


\section{Cet article analyse la signification socio-juridique des performances} transgressives réalisées par Piotr Pavlenskiy en Russie (2012-2015) et à Paris (2017), où il a obtenu l'asile politique en 2016. Si elles ont eu des retombées légales en France comme en Russie, l'ensemble de ces actions n'y ont pas cependant donné lieu au même type de débat. Pourquoi cet art a-t-il été accueilli différemment en Russie et en France? La première partie de l'article se penche sur les ressorts de la popularité de Pavlenskiy en Russie, et propose de considérer ses performances non pas uniquement comme étant iconoclastes, mais aussi comme des formes anti-hégémoniques d'énonciation de la loi. La seconde partie analyse la trajectoire parisienne de l'artiste, et avance des hypothèses pour expliquer les raisons du relatif «échec» de sa stratégie artistique dans le contexte socio-politique et juridique français.

«L'artiste performeur russe Piotr Pavlenskiy a été arrêté par la police, lundi 16 octobre à l'aube, pour avoir mis le feu à une antenne de la Banque de France, place de la Bastille à Paris. Peu après 4 heures du matin, l'artiste a aspergé d'essence deux fenêtres encadrant l'entrée du bâtiment, puis y a mis le feu. L'artiste et sa compagne Oxana ont été arrêtés et placés en garde à vue pour dégradations volontaires de biens par l'effet d'une substance incendiaire», pouvait-on lire dans un bref article du Monde, datant du 16 octobre 2017 1. L'article annonçait Éclairage, la première action parisienne de l'artiste, qui avait fui la Russie pour la France, où il avait obtenu l'asile politique six mois auparavant.

En Russie, la brève carrière de personnalité publique de Piotr, jeune artiste saint-pétersbourgeois quasi autodidacte, comptait plusieurs performances qui ont marqué les esprits par leur aspect provocateur et subversif. En 2012, il s'est cousu la bouche au fil rouge pour affirmer son soutien aux Pussy Riot récemment arrêtées, s'est enveloppé tout nu dans du fil barbelé sur les marches d'un bâtiment gouvernemental à Saint-Pétersbourg, puis est allé jusqu'à se clouer les testicules aux pavés de la place Rouge. II ne s'en est pas tenu là et s'est par la suite coupé une partie de l'oreille droite sur le toit de l'hôpital psychiatrique Serbsky à Moscou, a plus tard simulé un incendie au centre de Saint-Pétersbourg, rappelant I'insurrection sur la place Maidan-Nezalezhnosti à Kiev, à laquelle il avait participé en 2014. Enfin, il plaqua l'accord final en ciblant la porte de la fameuse
«Loubianka» - siège historique du FSB, successeur direct du KGB à Moscou².

Tout comme à Paris, l'artiste avait donc mis le feu à un monument à forte valeur symbolique - ou plutô† «avait fortement endommagé le vernis de la porte d'entrée d'un bâtiment administratif », comme le décrit l'enquête pénale, engagée lors de son procès. En effet, I'examen juridique de ses performances en Russie n'a jamais été uniquement un accompagnement, ou une conséquence indésirable de sa démarche provocatrice, mais constituait au contraire sa finalité. La méthode artistique développée par Pavlenskiy entre 2012 et 2017 peut se comprendre comme une sorte d'enquête expérimentale sur le fonctionnement des mécanismes punitifs du droit pénal russe. Une enquête artistique et personnelle tout aussi invraisemblable que risquée, où le protagoniste acceptait volontairement de prendre part à toutes les étapes du processus judiciaire déclenché par ses actions dans l'espace public, en les intégrant à la dramaturgie de son œuvre. Pourtant, malgré des accusations de plus en plus sérieuses, Pavlenskiy réussissait toujours à tirer son épingle du jeu, en évitant le carcan punitif russe, tel un Houdini. Cette prouesse, à la fois spectaculaire et improbable, a probablement été l'élément moteur de sa notoriété artistique dans l'espace postsoviétique.

À première vue, on pourrait croire que l'«entrée en scène» à Paris de Pavlenskiy est une réplique exacte de sa performance précédente, l'artiste recourant ici au principe de l'autocitation, voire de l'autoparodie:
1. «L'artiste russe Pavlenski arrêté à Paris pour avoir mis le feu à la Banque de France» [en ligne], disponible sur: https://www.lemonde.fr/ culture/article/2017/10/16/ l-artiste-russe-pavlenskiarrete-a-paris-apres-avoirmis-le-feu-a-la-banque-defrance_5201602_3246.html (consulté le 26 mai 2020)

2. À l'heure actuelle l'artiste a réalisé sept actions, nommées respectivement Suture (2012), Carcasse (2013), Fixation (2014), Séparation (2014), Liberté (2014), Menace (2015), Éclairage (2017) 
3. Pour une analyse de la place des groupements artistiques au sein des mouvements politiques russes, voir Johnson 2015 ; Gabowitsch 2016; Beumers et al. 2017.

4. Ainsi Pavlenskiy a-t-il été désigné deux fois comme l'artiste le plus influent de la scène de l'art contemporain russe parl'importante revue russe Artguide (en 2013 et en 2015). En 2010, le group Voïna, pratiquant la performance politique en marge de la légalité, s'était vu décerner le prix national Innovatsia, auquell'artiste a également été nominé. certains critiques russes qui se sont exprimés sur le sujet sont même allés jusqu'à dire qu'il s'était «essoufflé», qu'il se répétait.

Et si confronter un même geste artistique à deux systèmes sociojuridiques différents avait été guidé par un désir d'expérimentation presque scientifique? C'est ainsi en tout cas que Pavlenskiy définit lui-même sa démarche. II est vrai que cette nouvelle mise en scène à Paris intégrait tous les éléments nécessaires à la réussite d'une expérience comparative. Il y avait une «constante»: I'artiste lui-même; un nombre de variables indépendantes: un nouveau milieu artistique, un nouveau système juridique, une configuration médiatique différente; enfin, une variable dépendante: les conséquences, notamment juridiques, de sa performance. En mettant le feu à l'entrée de la Banque de France, Pavlenskiy semblait être hanté par un tout nouveau questionnement, qui pourrait se résumer en une simple phrase: son art sera-t-il reçu différemment à Paris qu'à Moscou?

Cette recherche entamée par l'artiste pourrait - par l'ensemble de données empiriques qu'elle génère, comme par son ouverture aux hypothèses heuristiques - intéresser l'anthropologie politique et juridique, qui ne s'ouvre pourtant que très épisodiquement aux questionnements liés au monde de l'art. En fin de compte, la méthode artistique élaborée par Pavlenskiy n'est pas si éloignée des formes les plus immersives de l'observation participante, et de ce fait, s'apparente à l'étude ethnographique des systèmes juridiques. Pourtant, contrairement à celle de l'ethnographe, la finalité de l'œuvre de l'artiste performeur n'est pas de décrire exhaustivement la nature d'un système juridique, mais de la représenter de manière tropique, voire d'incarner - au sens de leur faire prendre corps - les conflits et les tensions sous-jacents, opposant ledit système à une partie de la société.

\section{L'ART POLITIQUE EN RUSSIE}

Pour tenter de donner une réponse, ou, à défaut, un commentaire socioanthropologique nuancé à la question posée par l'artiste, il est important de rappeler quelques éléments sur le contexte socioculturel où a émergé l'œuvre de Piotr Pavlenskiy, et dans lequel elle était inscrite jusqu'à il y a peu, ainsi que le déroulement de ses actions clés.

Contrairement à la France, la Russie contemporaine est rarement imaginée comme une société où le débat et la contestation politique joueraient un rôle important. Bien au contraire: le maintien au pouvoir d'un président unique, sa politique extérieure militariste, le système juridique dominé par l'État, ainsi que le rejet systématique des normes du droit international, relayés par les grands médias occidentaux, font de la Russie, de plus en plus, un exemple d'«autoritarisme pur», rappelant son prédécesseur, I'URSS.

Pourtant, malgré la continuité des hégémonies, les contre-pouvoirs ne sont pas absents de la vie politique russe. Son paysage reste défini par une forte tension entre les forces pro-gouvernementales, qui dominent majoritairement la sphère parlementaire et publique, et celles de l'opposition non parlementaire, se confrontant au régime actuel par des moyens très réduits. Le réseau d'opposition est vaste, composé en grande partie (mais pas uniquement) d'individus hautement éduqués, mais dont l'expression est restreinte à une existence éphémère, reléguée aux plateformes de revues numériques et aux réseaux sociaux, sans compter les quelques manifestations de rue, de plus en plus difficiles à organiser et de surcroît violemment réprimées.

Dans cette configuration asymétrique, où le journalisme d'investigation continue d'être persécuté, la soif d'espaces permettant la circulation de discours alternatifs devient palpable. Dans ce système, la production intellectuelle en général et l'art visuel en particulier occupent une place à part entière, comparable à une sphère publique alternative. Au cours des vingt dernières années, tout un courant artistique, généralement appelé polititcheskoe iskusstvo (art politique) ou polititcheskiy aktionnism (l'actionnisme politique), a pris forme, mêlant les stratégies de l'art contemporain à celles de l'activisme de rue $\mathbf{3}^{\mathbf{3}}$.

Grâce à l'intérêt des médias occidentaux, on se souvient forcément du groupe ukrainien FEMEN, protestant seins nus contre l'ordre du jour patriarcal, en Ukraine puis en Europe, ainsi que du procès des Pussy Riot, jeunes femmes punk suppliant la Sainte Vierge de chasser Poutine lors d'une messe ironique à la cathédrale du Saint-Sauveur à Moscou, ou du corps dénudé de Piotr Pavlenskiy, s'exhibant en silence s ur la place Rouge. II semblerait que des expressions artistiques dites «radicales», associant la nudité, I'automutilation ou la dégradation des biens publics et confinées dans les sociétés occidentales au monde de l'art dont elles dépassent rarement les frontières tout en y occupant une position marginale, bénéficient en Russie d'une attention publique plus accrue ${ }^{\mathbf{4}}$.

On pourrait supposer, d'une part, que ces gestes iconoclastes comme les images qui en découlent, véhiculées par la presse, rassemblent métaphoriquement l'éclectisme des opinions politiques de l'opposition, tout en incarnant une série d'états émotionnels - tels la colère, l'abattement ou le désarroi face à la vie politique - reconnus et partagés par un grand nombre de spectateurs; et d'autre part, que ces actions artistiques ont une fonction révélatrice: à la frontière entre le monde symbolique et le monde réel, elles ouvrent des espaces de débat, de confrontation, voire de scandale, mettant en évidence les clivages entre les opinions progressistes et conservatrices, qui dépassent de loin les milieux des amateurs d'art. Les actions d'artistes - performances radicales bousculant les règles de la sphère publique - contribuent en quelque sorte à la formation d'un mode d'expression alternatif, provocateur ou ironique à souhait, qui échappe au storytelling des médias progouvernementaux. 


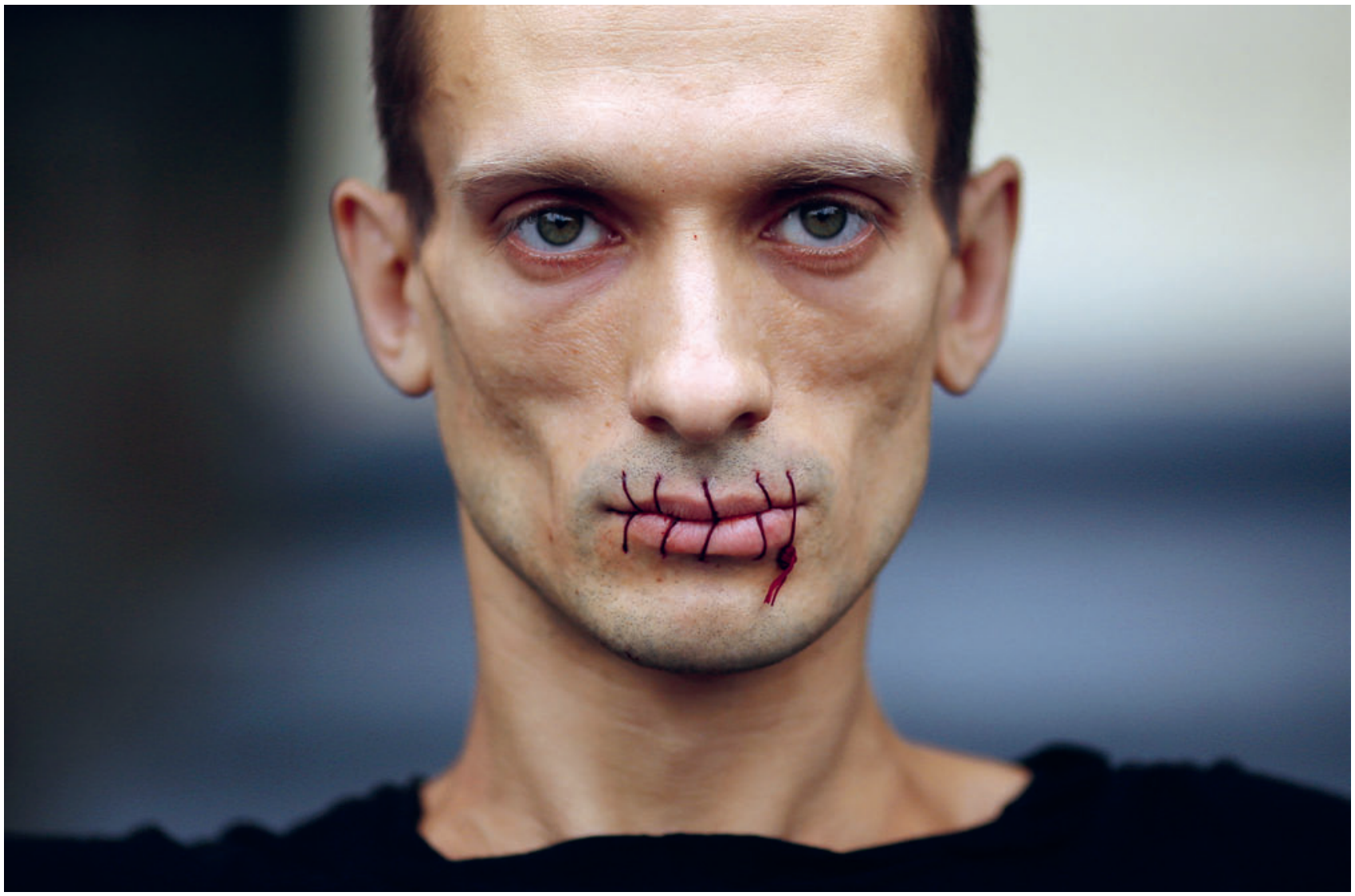

Piotr Pavlenski la bouche cousue, en soutien aux Pussy Riot et contre la censure, le 23 juillet 2012 à Saint-Pétersbourg. Avec l'aimable autorisation de l'artiste. DR.

\section{PIOTR PAVLENSKIY, ARTISTE POLITIQUE OU «JURIDIQUE »?}

Les performances de Piotr Pavlenskiy, toujours impromptues (seuls des journalistes choisis sont avertis quelques heures avant l'événement du contenu de la performance), visent en premier lieu les témoins oculaires aléatoires, mais surtout les agents de l'ordre public - gardiens, policiers, parfois médecins.

C'est bien leurs réactions immédiates, caractérisées par une désapprobation ostentatoire quasi instinctive, qui vont jouer dans les performances de Pavlenskiy un rôle dramaturgique décisif. Selon les circonstances, mais aussi la conception de l'artiste, qui «délègue» l'usage de son corps au pouvoir, il se retrouve transporté dans des lieux disciplinaires divers. Le plus souvent au commissariat de police ou bien à l'hôpital psychiatrique, où l'artiste, demeuré silencieux sur la place publique, se met enfin à parler, dévoilant la signification de ses actions.

Ce dialogue entre l'artiste et l'institution correctionnelle se termine dans la plupart des cas par une mise en accusation, amenant le protagoniste à la salle du tribunal. Son geste artistique devient affaire juridique. Cette ligne droite, reliant la rue, la sphère médiatique, les espaces carcéraux, I'hôpital psychiatrique et le tribunal, constitue pour ainsi dire la signature personnelle de Piotr Pavlenskiy. Si elle est personnelle, elle n'est cependant pas singulière: elle s'inspire directement des trajectoires d'un grand nombre d'individus, ayant protesté contre leur condition, affronté les autorités pour finir au procès.
Notons que cette dernière étape, où les actions de l'artiste sont mises à plat et débattues devant un juge, bénéficie d'une grande publicité - en Russie, tous les procès sont ouverts, et la performance devient spectacle judiciaire. Le rythme des assises alimente le suspense, retient l'attention du public, avant cette sorte de catharsis qu'est la décision finale. Si elle est en faveur de l'artiste, on s'en félicite et célèbre une «victoire», au moins provisoire, dans le cas contraire, on relance le débat sur l'état lamentable du système juridique russe. Ainsi, il serait réducteur de ne percevoir l'œuvre de Pavlenskiy que comme une succession d'images parlantes, issues de performances transgressives et scandaleuses; il s'agit plutôt d'un ensemble dramaturgique, basé sur une alternance d'épisodes narratifs - visuels, artistiques, médiatiques et surtout judiciaires.

Les performances de Pavlenskiy pourraient donc être reconstituées non seulement à partir des reportages et images médiatiques, mais également, dans un tout autre registre narratif, par la production bureaucratique qui les accompagne - procès-verbaux des assises, des inculpations du procureur, des tirades défensives des avocats et des décisions des juges. Au cours des cinq années de sa carrière russe, les activités artistiques de Pavlesnkiy ont pris la forme de plus de dix tomes de divers documents juridiques, où celles-ci sont interprétées, dans l'ordre chronologique, comme des «actes de hooliganisme mineur $\mathbf{5}_{\text {» }}$ - concept du droit administratif soviétique et postsoviétique, équivalent approximatif de la catégorie occidentale de «l'infraction à l'ordre public», puis comme
5. Art.20-1 du Code des contraventions administratives de la Fédération russe. 
6. Art.213-1-B du Code péna de la Fédération russe.

7. Art.20-2 du Code des contraventions administrative de la Fédération russe.

8. Art.214-2 du Code pénal de la Fédération russe.

9. Art.322-1 du Code pénal français.

10. Les notions de hegemonic et counter-hegemonic [legal] storytelling peuvent être apparentées, respectivement, au «storytelling politique introduit en France par introduit en rance par Christian Salmon, ainsi qu'aux "contre-fictions», développée par Yves Citton à partir des exemples du domaine littérair et artistique (Salmon 2013; Citton 2012). Outre le fait que Citton et Salmon n'analysent Citto pas le fonctionnement des narrations dans le contexte juridique, les modèles théoriques d'Ewick et Silbey sont, de manière générale, plus universels. Ne se résumant pas aux modes d'expression pas aux modes d'expression
résistante, propres aux milieux résistante, propres aux milie
culturels, ils permettent notamment de tracer des continuités entre les gestes artistiques et ceux de la vie ordinaire. «actes de hooliganisme liés à la haine religieuse ou politique envers un groupe social $\mathbf{6}_{\gg\rangle}$ (délit similaire, mais relevant du droit pénal), puis comme des cas d'infraction aux «règles d'organisation de manifesta-

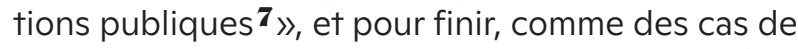
«vandalisme lié à la haine religieuse ou sociale $\mathbf{8}_{\text {», }}$ selon la formule juridique russe, ou de «destruction, dégradation ou détérioration d'un bien appartenant à autrui ${ }^{9} \gg$, selon la française.

Traduites en langage des chiffres, les œuvres de Piotr, qui n'ont jamais franchi le seuil des galeries, ont tout de même vu leur «prix» monter significativement: les amendes souscrites par le tribunal sont passées de 50 roubles (approximativement 0,70 euro) en juillet 2012 à 500000 roubles en juin 2016 (approximativement 7000 euros). Le tribunal correctionnel de Paris a condamné Pavlenskiy et sa compagne à payer à la Banque de France 3000 euros de dédommagement moral et 18 678,14 euros de dédommagement matériel. Il en va de même pour la notoriété de l'artiste: jeune inconnu en 2012, il acquiert une grande notoriété en moins de deux ans, malgré son œuvre plus qu'éphémère et son refus de «solidifier» son geste en une œuvre d'art commercialisable représentée par une galerie ou un agent.

\section{ÉLÉMENTS DE COMPRÉHENSION DE LA NOTORIÉTÉ DE L'ARTISTE EN RUSSIE: DES ACTIONS CONTRE-HÉGÉMONIQUES À LA CONSTRUCTION D'UNE NARRATION JURIDIQUE SUBVERSIVE}

Soulignons d'abord que ces œuvres sont conçues par son auteur non pas comme des «objets d'art» figés dans l'espace, mais comme des événements, mettant à l'épreuve les règles définies de l'expression artistique et publique. Autrement dit, dès leur conception, elles appartiennent simultanément au champ de l'art, en tant qu'œuvres esthétiques à part entière, et au champ juridique, où elles deviennent des cas, souvent à forte composante politique, débattus au sein de la sphère médiatique locale et internationale. Par cette double, voire triple appartenance (artistique, médiatique et juridique), ces œuvres touchent un public inhabituel, transgressant les cloisonnements sociaux (et spatiaux) propres au public amateur de l'art contemporain. En effet, quand il s'agit de Pavlenskiy, les juristes s'intéressent volontiers à la performance nue, les journalistes politiques parlent des actes artistiques transgressifs, les représentants d'État mentionnent les organes génitaux de l'artiste dans leurs discours, le milieu artistique, quant à lui, déserte les galeries pour réserver des places dans les salles des tribunaux. La caractéristique centrale de ces performances, outre leur aspect formel, est leur capacité à bousculer et «mettre en mouvement» le public de l'art contemporain russe.

Le public cible de Pavlenskiy en Russie est celui qui reconnaît son geste comme étant «de l'art», tout en considérant ce genre de performance comme une affirmation politique, au même titre que toute autre manifestation publique d'opposants. On voit alors en action le principe métonymique de l'expression artistique: les péripéties que rencontre l'artiste - incarcéré, interrogé, jugé, puis libéré - face au dispositif punitif de l'État sont perçues par ses spectateurs comme une mise en procès de leur fantasme révolutionnaire commun, et donc vécues comme une expérience personnelle et directe.

Ainsi, l'artiste Piotr Pavlenskiy n'est pas seulement auteur de performances transgressives et d'images éloquentes reprises par la presse pour illustrer une situation politique. II se présente surtout comme narrateur, capable d'imaginer une fiction politique radicale, qui au-delà du champ de l'art, continue d'agir dans le champ juridique pour rendre visibles les structures hiérarchiques qui le régissent.

Selon les anthropologues Patricia Ewick et Susan Silbey, auteures de référence des études sociolégales anglo-saxonnes (Law and Society Studies), les narrations sont des actes sociaux produits par - mais aussi constitutifs - des contextes spécifiques (historiques, institutionnels et interactionnels) et qui organisent et normalisent le sens, comme les conséquences de ces actes. Ainsi, les narrations juridiques - autrement dit, les manières de raconter les interactions entre I'individu et le droit - sont, comme tout autre discours, contraintes par «les règles de performance» (rules of performance) et obéissent à «des normes de contenu» (norms of content), dictées par le contexte dans lequel lesdits discours s'inscrivent. En répondant - pleinement ou partiellement - à ce dispositif réglementaire, des narrations juridiques peuvent contribuer à la cristallisation et à la reproduction d'une «narration hégémonique» (hegemonic storytelling), propre à chaque système juridique (Ewick et Silbey 1995; 2003).

Mais, toujours selon Ewick et Silbey, les narrations peuvent également jouer le rôle inverse, participant à la déconstruction de l'hégémonie ou à sa transformation idéologique. On parle alors d'«histoires subversives» (subversive stories), qui, en contournant les règles de l'énonciation ou en désobéissant aux normes du contenu, esquissent la possibilité (factuelle ou fictionnelle) d'une histoire alternative - soit une suite d'événements qui ne répond pas à l'ordre hégémonique des choses prédéfini. On évoque alors la possibilité d'une «narration juridique contre-hégémonique» (counter-hegemonic legal storytelling 10). Le cas Pavlenskiy, du moins en Russie, fait indéniablement partie de ceux-là. Son histoire en Russie était, jusqu'à il y a peu, celle d'une victoire symbolique obtenue dans un combat asymétrique contre les instances judiciaires elles-mêmes, une success story, inversant les relations entre forts et faibles, le tout dans un contexte historique et politique où de telles opérations semblent pratiquement impossibles. 
Quand l'art fait loi: histoires juridiques de Piotr Pavlenskiy en Russie et en France Nataliya Tchermalykh

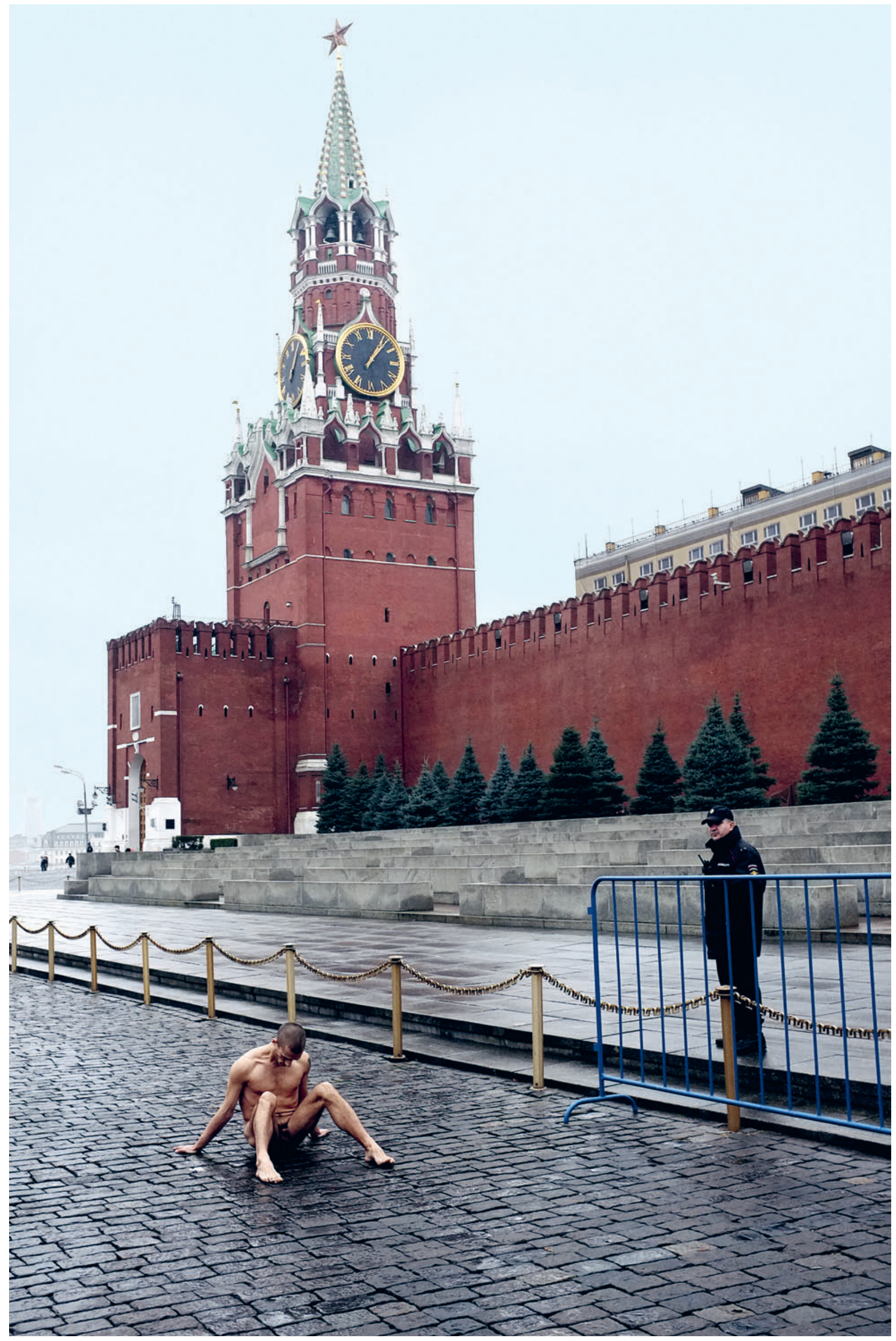

Fixation, performance réalisée le 10 novembre 2013, à l'occasion de la journée nationale de la police, en face du mausolée de Lénine sur la place Rouge à Moscou. Avec l'aimable autorisation de l'artiste. DR. 


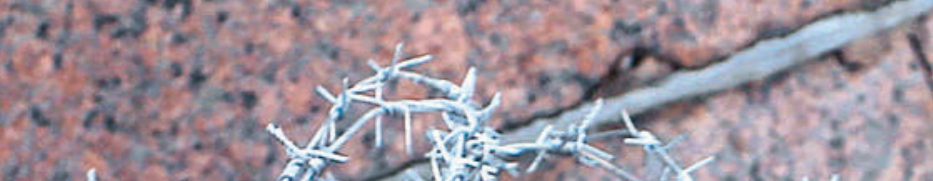

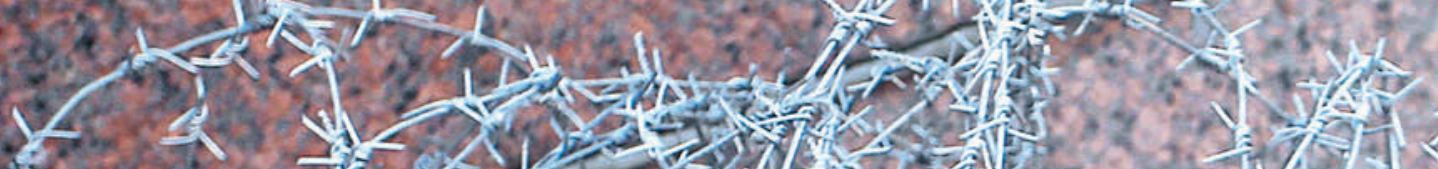
- 1 -

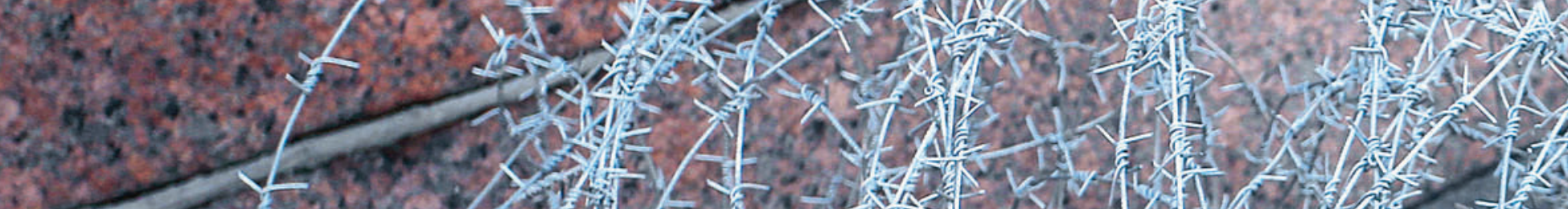
$=(-1)$ $=7 \times$ \&

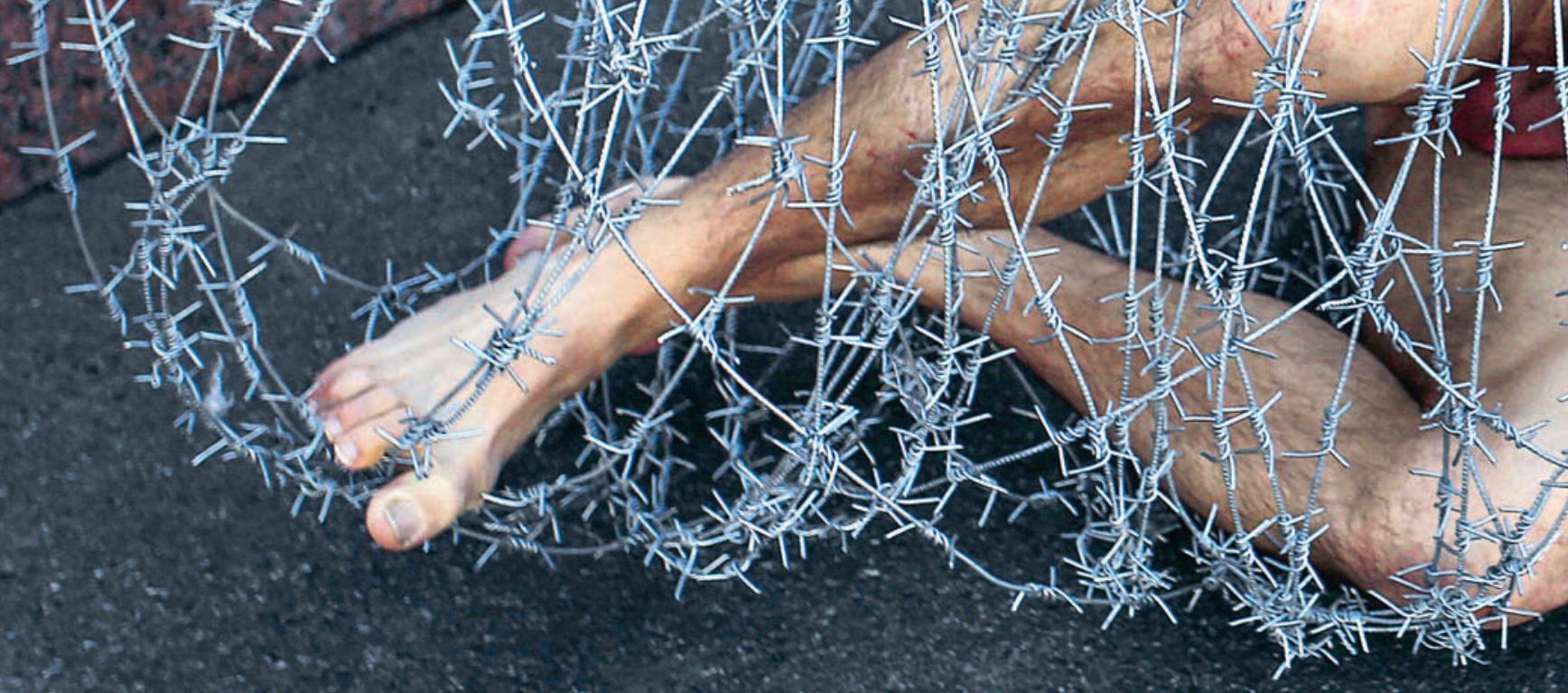


wat

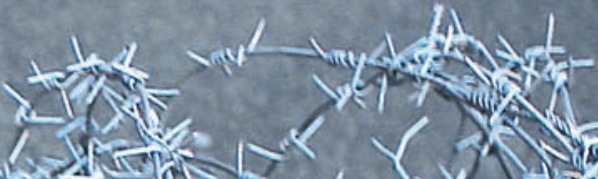

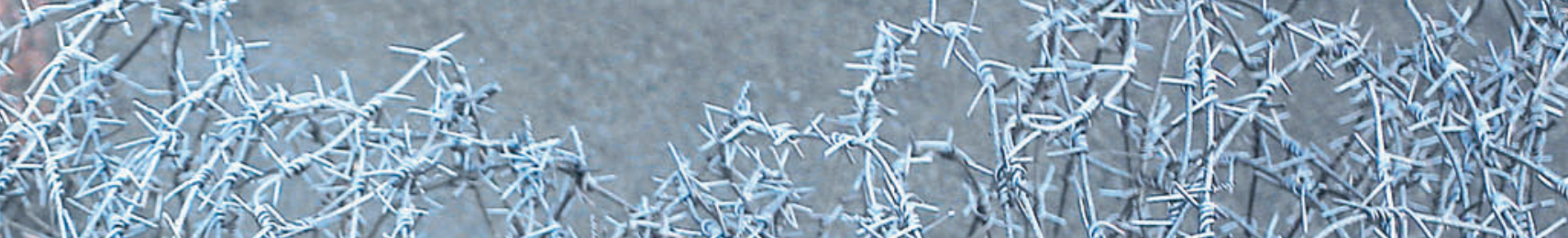

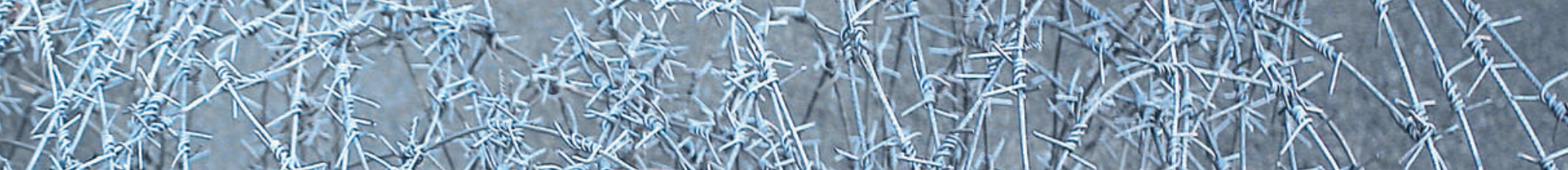

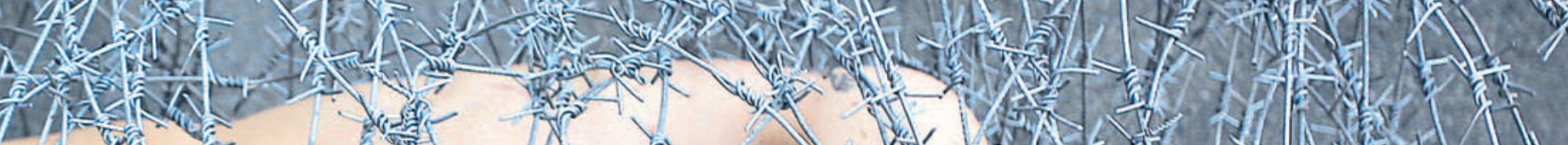
40

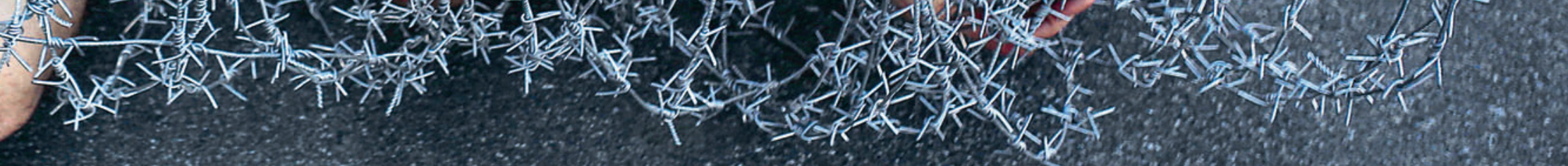




\section{Études et essais}

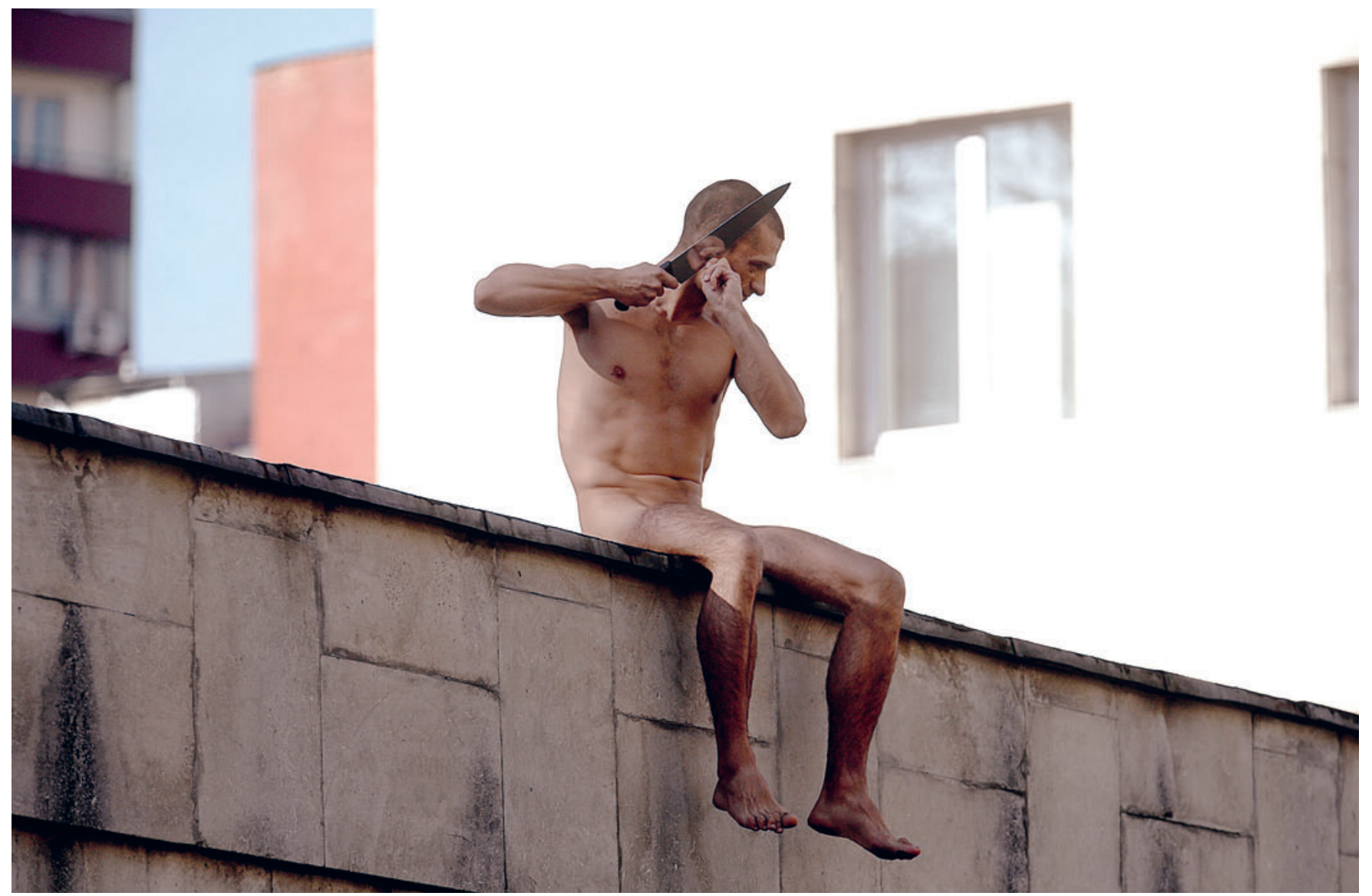

Segregation, sur le mur d'enceinte de l'institut psychiatrique Serbski, à Moscou, 21 octobre 2014. Avec l'aimable autorisation de l'artiste. DR.

11. Art.19-34 du Code des contraventions administratives de la Fédération de Russie.

\section{ALLIANCES IMPROBABLES ENTRE L'ARTISTE}

ET LES PROFESSIONNELS DU DROIT: UNE HISTOIRE SUBVERSIVE?

D'un point de vue rationnel, la démarche de Pavlenskiy ne peut que sembler «suicidaire» juridiquement parlant - il est difficile de croire en effet qu'un acte tel que l'incendie de la porte d'entrée d'un bâtiment public, appartenant de surcroît aux services secrets, puisse connaître un quelconque dénouement alternatif ou «contre-hégémonique». E† pourtant, Piotr Pavlenskiy, «artiste juridique» de longue date, en choisissant de mettre le feu au centre de sa performance, s'essayait dans le rôle du metteur en scène de son propre procès et espérait gagner.

Au petit matin du 9 novembre 2015, Piotr était descendu d'un train de nuit reliant Saint-Pétersbourg à Moscou, muni d'un bidon d'essence. À la gare, il a commandé un taxi jusqu'au numéro 20 de la rue Bolshaya-Lubyanka, où se trouve le bâtiment historique du FSB, acronyme désignant les services de renseignement de la Fédération russe. Les journalistes avertis étaient déjà là. Arrivé devant l'entrée principale, Piotr a aspergé d'essence les boiseries de la porte avant d'y mettre le feu avec un briquet. Quand la porte s'est mise à flamber, l'artiste s'est retourné un instant pour poser face aux quelques photographes présents, puis s'est rendu à la police de son plein gré.
Prendre le dessus sur le déroulement d'un procès, en contournant aussi ingénieusement que possible son dispositif réglementaire: voilà le mot d'ordre et la stratégie favorite de Pavlenskiy. Cette dernière s'apparente à celle des activistes, utilisant le tribunal comme une arène politique et une scène d'expression - stratégie largement utilisée par les militants et les intellectuels engagés depuis le début du $x x^{e}$ siècle (Israël 2009). À ceci près que l'objectif final de Pavlenskiy, malgré ses accents politiques, reste celui du geste artistique. Pour cela, il choisit de «jouer avec le feu» - au sens propre comme au figuré - en explorant les formes d'actions habituellement réservées aux insiders du champ juridique, aux professionnels de droit.

Dans cette optique, Piotr Pavlenskiy n'a pas agi seul: dès ses débuts, il est soutenu par les avocats du groupe AGORA, association informelle d'avocats russes politiquement engagés. Par le passé, ils avaient dû abandonner leur ONG basée à Kazan (République de Tatarstan) spécialisée dans la défense des victimes des forces de l'ordre et des prisonniers politiques. Cette organisation a compté parmi les premières à être référencées par le ministère de la Justice russe en tant qu'«agent étranger» (inostrannyi aguent), néologisme juridique repris en 2005 pour cibler toute association subventionnée par des organisations étrangères ou internationales 11 . Depuis, ces avocats exercent leur métier en indépendants, défendant à titre gracieux les causes dont ils se sentent proches. Parmi eux, quatre ont travaillé avec Pavlenskiy, devenant ses «guides» dans le champ juridique. 


\section{Quand l'art fait loi : histoires juridiques de Piotr Pavlenskiy en Russie et en France}

Nataliya Tchermalykh

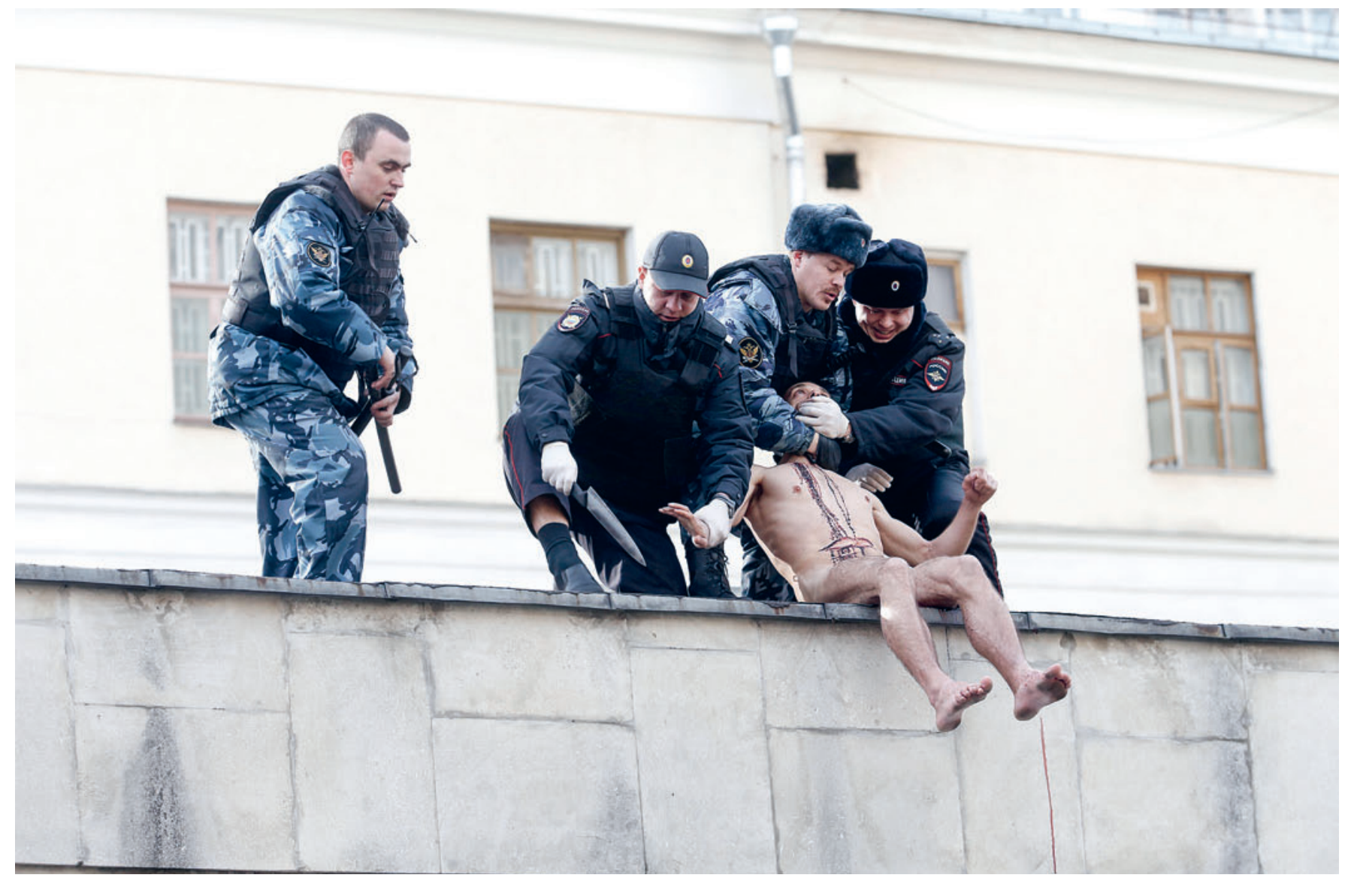

Segregation, sur le mur d'enceinte de l'institut psychiatrique Serbski, à Moscou, 21 octobre 2014. Avec l'aimable autorisation de l'artiste. DR.

Sans leur aide, son entreprise aurait probablement été vouée à l'échec. Pour la décrire, l'expression anglaise «turning tables» paraît plus adéquate que tout terme français. «Retourner les tables», c'est-à-dire, dans ce contexte, avoir recours à l'outil artistique et au savoir juridique de ses avocats pour renverser les dispositifs légaux conçus contre lui par le pouvoir. Puis retourner cette situation ironiquement et la réutiliser comme un épisode de plus dans sa dramaturgie complexe de la performance «augmentée».

Revenons par exemple sur un épisode qui a eu lieu lors de l'enquête autour de l'action Svoboda (Liberté) en 2014. II s'agit peu ou prou de la «conversion» d'un de ses enquêteurs, qui a démissionné pour entrer dans le «monde de l'art». Pavlenskiy en a tiré une pièce documentaire, intitulée Interrogatoire de Pavlenskiy. Une pièce en trois actes, basée sur les enregistrements audio des interrogatoires qui l'opposaient à l'enquêteur Pavel Yasman. La pièce a été publiée par snob.ru, site Internet très prisé par les milieux libéraux.

En voici un extrait, exemple éloquent de la transformation subtile des normes de l'interrogatoire, qui montre non seulement l'affrontement entre le monde de l'art et celui de la justice, mais également la façon dont le premier agit sur le dernier progressivement:

\section{[...]}

Enquêteur: Piotr Andréïevitch, mon cher, vous n'imaginez pas à quel point j'aime discuter avec vous. Mais comptez-vous rester longtemps ici? Vous savez que je suis au commissariat tous les jours, dès 7 heures du matin.

Pavlenskiy: Je voulais seulement tenter de trouver un terrain commun. Je voudrais comprendre la manière de fonctionner des deux côtés. La vôtre aussi, pas seulement la mienne. Mon «champ symbolique» ne correspond pas à votre «Code pénal».

Enquêteur: Qu'avez-vous dit? Je n'ai pas compris un mot. C'est un peu comme quand je lisais votre pétition, adressée au tribunal.

Pavlenskiy: J'ai pourtant essayé d'être aussi clair que possible en l'écrivant...

Enquêteur: Vous vous dites artiste. Comptez-vous aujourd'hui me montrer vos tableaux ou vos dessins? 
12. Допрос Петра Павленского Пьеса в трех действиях (Interrogatoire de Pavlenskiy. Une pièce en trois actes) [en liǵne], disponible sur : https:// snob.ru/selected/entry/77648/ page/2

(consulté le 7 janvier 2020). Traduit par l'auteure.

13. The Village [en ligne], disponible sur: https://www the-village.ru/village/city/ situation-comment/218341pavel-yasman

(consulté le 7 janvier 2020).

Traduit par l'auteure.
Pavlenskiy: Je n'en ai pas. Vous savez, ma spécialisation est très étroite.

Enquêteur: Laquelle?

Pavlenskiy: L'actionnisme.

Enquêteur: Alors je suis artiste, moi aussi!

Pavlenskiy: Seulement si vous réalisez des actions artistiques.

Enquêteur: Ça tombe bien. J'en ai réalisé quelques-unes aujourd'hui.

Pavlenskiy: Alors vous devez maintenant réfléchir dessus.

Enquêteur: J'ai déjà réfléchi. Beaucoup.

Pavlenskiy: Alors vous êtes déjà artiste.

Enquêteur: Je suis artiste?

Pavlenskiy: Bravo! Toutes mes félicitations!

Enquêteur: Je suis artiste, enquêteur du département central de l'investigation criminelle de la ville de Saint-Pétersbourg. Artiste de Justice.

Pavlenskiy: Vous pouvez même signer le protocole de l'interrogatoire... En tant qu'artiste $[. ..] \mathbf{1 2}$.

Ce dialogue aux accents absurdes, où le personnage de l'enquêteur, dont l'humour subtil correspond assez peu au profil type du policier russe, n'est pourtant pas tiré de l'adaptation théâtrale d'une œuvre de Gogol ou de Kafka - il ne s'agit pas non plus d'une approximation fictionnelle. Dans une interview que Pavel Yasman (I'enquêteur) nous a accordée en 2015, il a confirmé que ces échanges avec Pavlenskiy avaient vraiment eu lieu, et qu'ils avaient été décisifs dans sa trajectoire personnelle.

Depuis la publication de la pièce en ligne, où elle a été vue plus de soixante et onze mille fois, Pavel Yasman est devenu une sorte de figure publique des milieux libéraux moscovites et saint-pétersbourgeois. Dans un long entretien accordé à un journaliste de la presse indépendante, Yasman a révélé qu'il avait déjà entendu parler de Pavlenskiy avant et qu'il avait beaucoup hésité à se charger de l'affaire. On y apprend également quelques éléments biographiques: la performance de Pavlenskiy constituait en fait la première enquête de Yasman à Saint-Pétersbourg, où il avait été muté récemment, occupant précédemment le poste important de premier enquêteur de la ville à Voronezh. À Saint-Pétersbourg, il avait de nombreux désaccords avec sa hiérarchie, notamment sur la manière d'aborder le cas de Pavlenskiy. «Je n'ai rien contre cet homme. Je le trouve même humainement sympathique [...]. J'ai lu nos dialogues dans la presse avec plaisir, j'ai même beaucoup ri», a-†-il avoué, en poursuivant: «Mais en lisant les commentaires en ligne, j'ai vu que certains disaient que c'était moi, l'enquêteur de l'affaire, qui avait l'air d'un fou, mon honneur était un peu blessé $\mathbf{1 3}^{\mathbf{l}}$.» Cette mention de la folie n'est pas anodine: le procureur général avait en effet ordonné à Yasman de soumettre Pavlenskiy à plusieurs expertises psychiatriques, bien que la première l'eût reconnu sain. Ayant rencontré Pavlenskiy plus d'une fois, Yasman ne jugeait pas cette mesure nécessaire. Il avait même émis plusieurs requêtes pour éviter à Pavlenskiy d'affronter le corps psychiatrique. Le 7 août 2014, le tribunal a rejeté la requête de l'enquêteur une fois de plus. Le lendemain matin, Pavel Yasman a alors décidé de poser sa démission, quittant le comité d'enquête pour toujours. Six mois plus tard, il a réussi l'examen du barreau et est devenu avocat indépendant, rattaché au barreau de Saint-Pétersbourg. S'il n'est pas devenu «artiste de justice», comme le prédisait Pavlenskiy, il a cependant opté pour la relative indépendance encore procurée par le statut d'avocat en Russie.

Les rebondissements inattendus de cet épisode ne se sont pas arrêtés là: lors des assises suivantes, Pavlenskiy a surpris son auditoire en annonçant que Pavel Yasman - qui venait de démissionner - allait devenir son défenseur public. L'artiste avait même rédigé une requête officielle auprès du tribunal, qui avait été, bien évidemment, refusée par le juge. Cette fois, le geste artistique en restait au stade de la provocation symbolique.

\section{LA SOLIDARITÉ POLITIQUE AU-DELÀ DES CLOISONNEMENTS SOCIOPROFESSIONNELS: PIOTR PAVLENSKIY ET LA CAUSE DES 《TERRORISTES DE CRIMÉE 》}

Celui-ci n'est qu'un épisode parmi de nombreux autres permettant à Pavlenskiy de s'affirmer auprès du tribunal en tant que sujet à l'avant-garde de sa propre défense, en contribuant pleinement, voire en élaborant sa stratégie défensive, même s'il se distingue par son côté ironique et bienveillant; d'autres étaient en effet plus sombres ou radicaux. Le début du procès autour d'Ugroza (Menace), la performance aux portes de la FSB, était marqué par un geste très inhabituel: Pavlenskiy, accusé d'«offense à l'ordre public», d'«attitude haineuse», de «vandalisme», puis d'«endommagement d'un objet d'héritage culturel», avait demandé au tribunal de disqualifier son accusation selon l'article 205 du Code pénal russe traitant du «terrorisme».

II serait difficile de comprendre cette demande sans quelques éléments de contexte. Tout d'abord, les observateurs ont repéré une tendance générale chez la justice russe ces dernières années à disqualifier des cas d'opposition politique ouverte (allant des manifestations publiques à l'occupation ou mise à feu de bâtiments) relevant de l'article 213 du Code pénal 


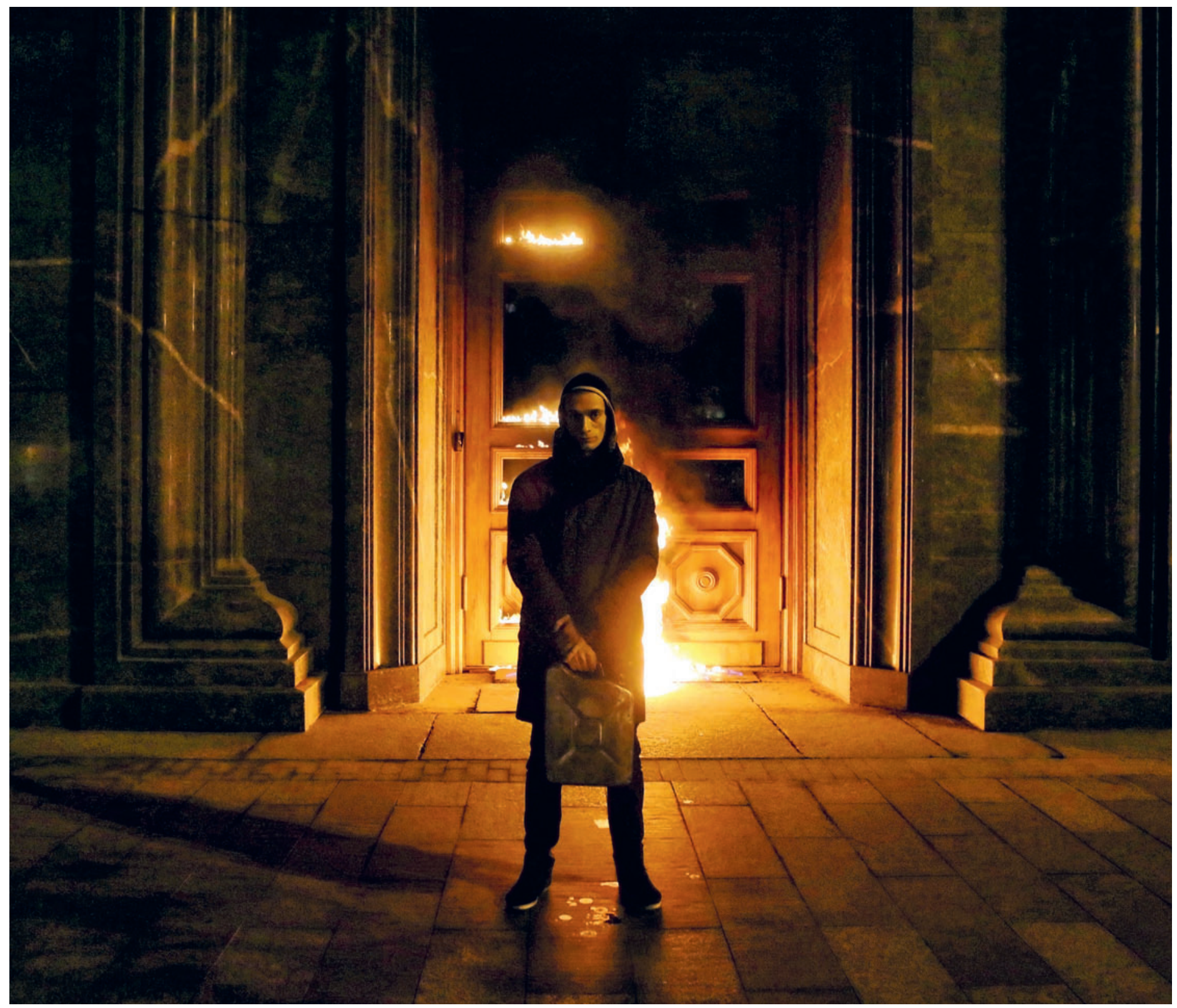

Threat, action à la Loubianka, siège des services secrets (Federal Security Service / FSB), Moscou, 9 novembre 2015. Avec l'aimable autorisation de l'artiste. DR.

(«infraction contre l'ordre public» ou «hooliganisme») par recours à la législation contre «l'extrémisme» ou le «terrorisme», les participants de groupes d'opposition étant inculpés pour «formation de groupuscules terroristes dans le but de réaliser des actes visant les populations civiles» (article 205 du Code pénal), même si ces actes sont inexistants. Ainsi, selon les données statistiques provenant du département judiciaire de la Cour suprême de la Fédération de Russie, en 2015, cinq cent quarante-quatre personnes ont été reconnues coupables d'activités extrémistes ou incitant à l'extrémisme, cent dix autres ont été inculpées pour terrorisme ou incitation au terrorisme. Par rapport à 2014, les chiffres ont augmenté de plus de $25 \%$.

Parmi les derniers grands procès en date, les cas les plus éloquents concernent l'opposition antirusse en Crimée - territoire ukrainien annexé par la Fédération de Russie en mars 2014 et immédiatement entré sous juridiction russe. Entre 2014 et 2019, quelques dizaines de procès antiterroristes contre des individus ou des organisations politiques s'opposant au nouveau régime ont eu lieu dans les tribunaux de la péninsule, désignant particulièrement deux groupes sociaux: les activistes politiques pro-Ukrainiens, ne reconnaissant en aucun cas l'annexion de la Crimée; et les Tatars de Crimée, population autochtone et majoritairement musulmane, historiquement loyale à la souveraineté ukrainienne. Parmi les accusés, aucun n'a été acquitté. Le geste de Pavlenskiy était une manière de contester le procès d'Oleg Sentsov, premier de la série dite «des terroristes criméens». Sentsov, jeune réalisateur documentariste ukrainien, avait été condamné par un tribunal militaire russe (toute requête d'extradition en Ukraine lui ayant été refusée) à vingt-cing ans de prison ferme pour «organisation d'un groupuscule terroriste» et «préparation d'un

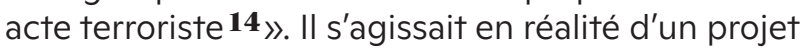
de destruction d'une statue de Lénine à Sébastopol, qui n'a jamais eu lieu. Le procès de Sentsov
14. Oleg Sentsov a été libéré lors d'un échange de prisonnier entre l'Ukraine et la Russie le 7 septembre 2019. 
15. En France, la cause d'Oleg Sentsov était soutenue par l'association Les Nouveaux dissidents, fondée en 2016 par Michel Eltchaninoff (https:// www lesnouveauxdissidents. org/). Des personnalités org/). Des personnalités
publiques comme Jonathan Littell, Emmanuel Carrère ou Christiane Taubira ont manifesté leur soutien au cinéaste emprisonné

16. Il s'agit du Cas Pavlenski: la politique comme art, Paris, Louison Éditions, 2016. est aujourd'hui cité comme le plus criant des exemples récents de «farce judiciaire» - procès ouvertement instrumentalisé à des fins politiques -, mais aussi d'assujettissement immédiat du territoire de Crimée au droit russe $\mathbf{1 5}$.

En dehors de leurs opinions politiques et de leur appartenance au monde de l'art, Sentsov et Pavlenskiy avaient un autre point commun: tous deux étaient défendus par Dmitri Dinze, avocat libéral bien connu à Saint-Pétersbourg et Moscou pour sa stratégie de «défense agressive» et ses interventions aux procès contre les tenants de l'opposition. De tous les avocats ayant défendu l'artiste, c'est avec Dinze que Pavlenskiy avoue avoir le plus d'affinités - tant sur le terrain des opinions politiques que sur celui des prises de risque stratégiques. Ensemble, ils formaient un tandem énergique et complice, transformant le tribunal en une arène de jeux rhétoriques acharnés. «Cette personne me comprend mieux que quiconque», nous a avoué Pavlenskiy lors d'un entretien en 2015. «Pavlenskiy, il est... comme moi!», a affirmé Dinze, rencontré à Saint-Pétersbourg le jour suivant.

Il est fort probable que les discussions avec son avocat, notamment autour des autres affaires de ce dernier, ont fait réfléchir Pavlenskiy et l'ont influencé dans sa démarche. La suggestion de requalification de son accusation en «terrorisme», adressée au parquet par Pavlenskiy, bien que pouvant, à première vue, être considérée comme une simple provocation «nihiliste», s'avérait en fait être un geste plus complexe. D'une part, il traduisait la solidarité de l'artiste avec d'autres clients de son avocat et sa sensibilité envers la «cause criméenne». D'autre part, cette «proposition » pointait avec netteté la tendance à l'utilisation punitive des accusations terroristes que seuls les professionnels du droit avaient jusque-là remarquée. Plus généralement, ce geste symbolique, inversant les rapports entre accusateur et accusé, signifiait une affirmation de pouvoir et d'«entrée en jeu» juridique de l'accusé.

Pour sa part, l'avocat Dinze n'a jamais restreint la marge de manœuvre de son client. Pour tout professionnel du droit en effet, il est évident que l'article employé dans le chef d'accusation ne peut être modifié à la demande de l'accusé, mais l'avocat semblait comprendre et partager pleinement la valeur symbolique des actes de l'artiste. «Les opposants criméens ont été inculpés de terrorisme pour un moindre mal. Je refuse de suivre vos rituels judiciaires si le tribunal ne m'écoute pas», a proclamé Pavlenskiy. Le juge a refusé, l'artiste s'est alors muré dans le silence et n'a pas dit un mot de tout le procès.

Malgré le silence du protagoniste, le procès a tout de même eu lieu, reposant sur d'autres acteurs: I'avocat Dmitri Dinze en premier lieu, sa femme Olga Dinze, également avocate, ainsi que les témoins de la défense, issus du monde de l'art et de la dissidence politique. Le comportement «irrespectueux» de l'accusé, qui ne s'est levé ni au début du procès ni au moment du verdict, et qui a refusé systématiquement tout échange verbal dans la salle, n'a pas aggravé son cas. Pavlenskiy a une fois de plus évité l'incarcération, bien qu'il ait été jugé coupable d'avoir «endommagé un objet de grande valeur culturelle» et condamné à une amende de 500000 roubles. Après avoir passé sept mois au centre de détention provisoire,

Pavlenskiy a donc quitté en homme libre la salle du tribunal Meschanskii à Moscou. Accueilli par des applaudissements à la sortie, il a dit en souriant: «Nous avons gagné.»

Lors des dépositions, l'avocat Dinze avait demandé au témoin à charge pourquoi il considérait la Loubianka (dont les souterrains sont associés aux tortures de la répression stalinienne) comme «objet de grande valeur culturelle». «Parce que beaucoup de poètes, d'artistes et d'écrivains ont passé du temps ici au cours de l'histoire!» a rétorqué le témoin, sans se rendre compte de l'aspect à la fois tragique et anecdotique de sa phrase. Cette formulation a par la suite été reprise telle quelle dans la décision officielle du juge. S'il fallait l'inventer, Pavlenskiy n'aurait pas pu faire mieux.

\section{DE L'ARTISTE CONTROVERSÉ AU DEMANDEUR D'ASILE: LE DÉTOUR RUSSO-FRANÇAIS D'UNE TRAJECTOIRE ARTISTIQUE}

Ainsi rapportée, la trajectoire russe de Pavlenskiy revêt un aspect très littéraire, son histoire se lit presque comme un roman sur la nouvelle dissidence russe, capable de déjouer, individuellement ou collectivement, les forces autoritaires, malgré les conditions défavorables. E† pourtant, la chute de son histoire relève bien moins du happy end qu'on ne pourrait le croire. Piotr Pavleskiy a pris la décision de quitter la Russie suite à une accusation d'agression sexuelle, déposée par une actrice d'un théâtre moscovite, Teatr.doc, où sa propre histoire était portée sur scène. L'affaire était d'autant plus compliquée qu'Oxana Shalyguina, la compagne de l'artiste, était également accusée d'agression. Tous deux ont nié les accusations avec vigueur, alléguant une manipulation. Cette fois, cependant, I'opinion publique russe ne pouvait être de leur côté.

Lors du premier interrogatoire, Piotr et Oxana, parents de deux petites filles, ont été avertis que s'ils venaient à être arrêtés au cours de l'enquête, compte tenu des antécédents criminels du père, les services sociaux engageraient une procédure de placement des enfants dans des institutions spécialisées. En présence de l'avocate de Pavlenskiy, l'enquêteur a tenu à préciser: «Mais pour l'instant, vous êtes libres.» Cette dernière a interprété cette phrase comme une invitation dissimulée à quitter le pays. Pavlenskiy, dont le recueil de textes et d'entretiens était en cours de traduction en français $\mathbf{1 6}$, a pris l'avion pour Paris le soir même. Les premiers mois, l'artiste et sa famille ont été accueillis et hébergés par son éditeur français. Trois mois plus tard, Pavlenskiy obtenait l'asile politique, et sa famille le droit de résider sur le territoire français. Pendant ce temps, à Moscou, la victime présumée ne s'est livrée à la presse qu'une seule fois, sans révéler de détails significatifs sur l'affaire; l'enquête n'a donc pas eu de suites, mais son délai 
de prescription à ce jour n'est pas échu. À l'heure actuelle, il est difficile de prendre position sur la véracité de cette accusation, mais l'artiste ne peut pas retourner en Russie sans repasser devant le tribunal.

On constate en revanche le refus de Pavlenskiy d'occuper la case idéal-typique de l'opposant à un régime autoritaire: en Russie déjà, il avait tendance à brouiller les schémas classificatoires de l'imaginaire politique collectif opposant les dissidents aux délinquants, les artistes et intellectuels aux «hooligans» et aux «vandales», bien que les deux groupes se retrouvent souvent côte à côte sur le banc des accusés. En 2015, Pavlenskiy avait ainsi choqué l'opinion publique libérale en annonçant son soutien au groupe Primorskiye Partisany (gang de sept jeunes hommes, réfugiés dans les forêts de la Taïga, qui menaient une guérilla armée contre les forces de l'ordre de la région de Primorié $\mathbf{1 7}$, dénonçant la corruption et la violence arbitraire de la police). Piotr Pavlenskiy s'apprêtait notamment à verser ses futures récompenses à une association assurant leur défense juridique. Cette décision lui a valu le prix Václav Havel18, auquel il a été nominé pour l'ensemble de ses performances. La décision de lui retirer le prix, prétextant la promotion de l'opposition violente par l'artiste, a été soutenue par l'opinion publique libérale en Russie.

Petit à petit, le portrait social de Pavlenskiy se complexifiait et s'obscurcissait, perdant les traits héroïques de l'incarnation parfaite du nouveau dissident. Bien qu'allant à l'encontre de son intérêt immédiat, ce brouillage de pistes semblait faire néanmoins partie de la stratégie personnelle de l'artiste, qui préférait une position marginale mais autonome à une case prédéterminée sur l'échiquier politique russe.

En arrivant en France, Pavlenskiy et sa compagne ont suivi la même ligne de conduite, aux accents nihilistes et antiétatistes, en refusant d'emblée toute aide financière, ainsi que l'aide au logement de l'Éta† français - choix stratégiques qui ont fortement conditionné leur trajectoire. Ne parlant qu'un anglais très rudimentaire au moment de leur arrivée, et ne maîtrisant pas les codes socioprofessionnels du milieu de l'art français, Pavlenskiy et sa famille se sont retrouvés confrontés à la marginalité, cherchant du soutien dans les milieux «antisystème» et ceux des squats. C'est dans ces conditions que Piotr Pavlenskiy a réalisé son entrée sur scène en France; son périple français et l'errance qui a suivi son arrestation, ainsi que le manque de reconnaissance du monde de l'art français témoignent des différentes manières dont l'art s'empare des questions politiques et juridiques dans les sociétés russes et françaises.

\section{L'INTRADUISIBILITÉ DES ICONOCLASMES? \\ L'ARTISTE RUSSE ET LE MONDE DE L'ART CONTEMPORAIN FRANÇAIS}

Malgré la reprise de la dépêche de l'AFP par la plupart des grands médias nationaux, annonçant l'action de l'artiste à Paris, l'affaire n'a pas suscité grand intérêt en France, y compris dans les milieux de l'art contemporain. Dans les mois qui ont suivi I'arrestation de Pavlenskiy, aucune revue d'art professionnelle n'a publié d'analyse de fond ou de débat autour du geste de l'artiste. Ainsi, Éclairage n'a suscité ni reconnaissance ni controverse, laissant en quelque sorte l'artiste russe «à la porte» du monde de l'art français, dont il semblait ne pas parvenir à franchir le seuil. Quelles sont les raisons d'une telle réticence, voire du rejet de son geste dans le contexte français?

Étant donné la singularité du cas, on ne peut qu'avancer des hypothèses, d'ordre à la fois culturel (relevant de l'intraduisibilité sémantique) et structurel (liées au contexte socioprofessionnel), pour expliquer cette disqualification.

Formellement, les deux dernières performances, Menace, qui visait la porte de Loubianka à Moscou, et Éclairage, qui visait celle de la Banque de France à Paris, rappellent Liberté, réalisée au petit matin du 22 février 2014 à Saint-Pétersbourg. Ce jour-là, Piotr Pavlenkskiy, accompagné de quelques militants anarchistes, a proposé une reconstitution (re-enactment) de l'esthétique des protestations ukrainiennes sur la place Maidan-Nezalezhnosti à Kiev, auxquelles il avait participé à I'hiver 2014. Mêlant les codes esthétiques du «tableau vivant» à ceux du photojournalisme sensationnaliste, l'artiste avait réuni tous les éléments de la réalité révolutionnaire ukrainienne: barricades de pneus en feu, fumée noire, drapeaux ukrainiens et anarchistes, hommes en cagoules, bruits de barres métalliques sur les pavés... Pendant quelques minutes et jusqu'à l'arrivée des pompiers et de la police, ce spectacle nocturne a paru bien réel. II l'était surtout grâce aux images, partagées massivement sur les réseaux sociaux.

L'idée de Piotr consistait à falsifier, pour ainsi dire, une réalité politique: les «preuves visuelles» qu'il a créées étaient situées dans un espace réel - en face de la cathédrale Vassilievski, sur le pont Malo-Konyushennyi, en plein centre historique de Saint-Pétersbourg, mais dans un temps fictif. C'était donc une action d'anticipation, qui se voulait performative - dans le sens linguistique plutôt qu'esthétique du terme.

Dans les performances qui suivront, il reprendra ce procédé en ayant recours à une forme d'iconoclasme métonymique, que Roland Barthes qualifiait de «sémioclasme» (Barthes 2015 [1957]). Celui-ci, basé sur le principe de pars pro toto, suppose que l'endommagement spectaculaire d'un élément isolé appartenant à un ensemble architectural représenterait symboliquement la résistance générale à une réalité politique associée dans l'imaginaire collectif audit ensemble. Un procédé qui n'est d'ailleurs pas uniquement artistique: il appartient en effet à la grammaire universelle des luttes populaires visant à délégitimer le pouvoir en place en s'attaquant à ses attributs $\mathbf{1 9}$. Pavlenskiy ne fait qu'emprunter et esthétiser ce geste, en lui accordant une valeur artistique.

Ainsi, à Moscou, Piotr Pavlenskiy avait choisi la porte de la Loubianka pour dénoncer l'influence du renseignement intérieur sur la vie politique russe. Ce choix faisait explicitement référence à la trajectoire
17. Le plus oriental des territoires russes, aux confins de la Chine, la Corée du Nord et du Japon.

18. Václav Havel Prize for Creative Dissent est décerné par la Human Rights Foundation, basée à New York La fondation est présidée par Garry Kasparov, champion du monde d'échees, puis homme politique russe et opposant

politique russe et opposant
à Vladimir Poutine. Kasparov est exilé aux États-Unis depuis 2012

19. Les historiens des mouvements révolutionnaires dressent tout un inventaire de destruction de symboles religieux et politiques par les foules à des moments de grands changements historiques, où « le rapport au visible se modifie, et les signes visuels du pouvoir deviennent sursignifiés» (Fureix 2014: 21). En 1789, on détruit massivement des effigies royales, avec leurs cortèges de mannequins brûlés ou pendus, de portraits fusillés ou renversés; en août 1792 ,

les statues des rois sont déboulonnées à la basilique de Saint-Denis, leurs corps exhumés et jetés à l'eau. En février 1848, la foule a brûlé le trône du roi Louis-Philippe, après l'avoir traîné à travers Paris. En 1870, après la destruction de la colonne Vendôme, de la maison de Thiers, l'incendie des Tuileries et de l'Hôtel de Ville, la Commune a laissé Paris en ruine. L'anthropologue Jack Goody observe dans son expérience personnelle la récurrence troublante des iconoclasmes populaires à l'échelle ólobale, au cours du $\mathrm{xx}^{\mathrm{e}}$ siècle: «J'étais en Italie en 1943 lors de la chute de Mussolini, quand les faisceaux furent arrachés des façades des monuments publies; en Allemaǵne éǵalement, lorsque Hitler tomba et croix gammées disparurent. Je me trouvais au Ghana en 1966 lorsque la chute de Nkrumah s'accompaǵna du renversement de sa statue devant le Parlement [...]. De nombreux changements moins violents ont entraîné le démantèlement de la statuaire publique de l'ordre précédent: la destruction à Bombay de la statue de la reine Victoria, la destruction de Staline en URSS...» (Goody 2006: 85) 


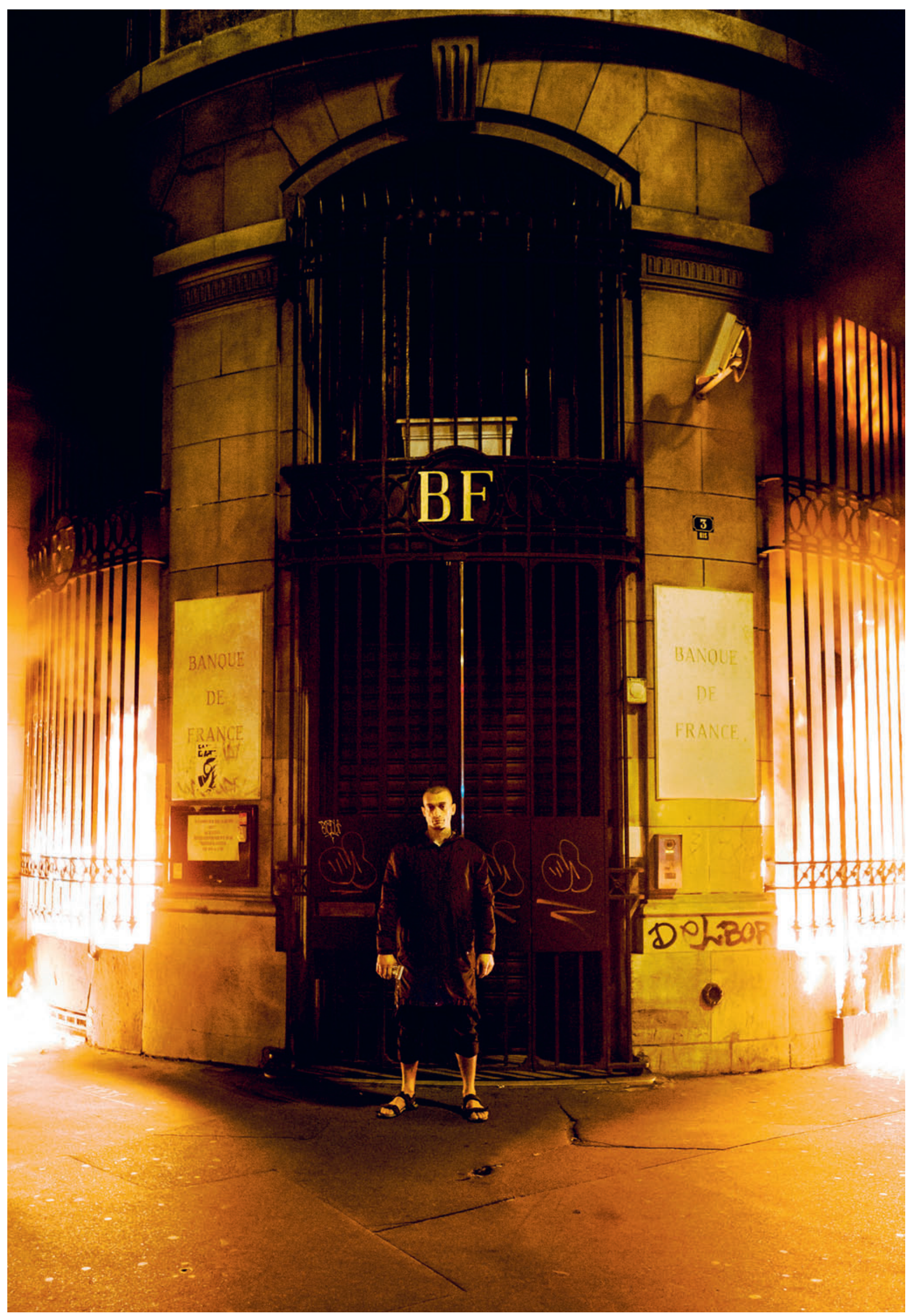

Lighting, action devant la Banque de France, Paris, 2017. Avec l'aimable autorisation de l'artiste. Photo Capucine Henry. 
professionnelle de l'actuel président, Vladimir Poutine, issu des services de renseignement. En s'attaquant au bâtiment de la Banque de France à Paris, l'artiste cherchait à mettre en lumière I'hégémonie du système financier et bancaire, auquel l'actuel président français a appartenu par le passé: «Nos contextes politiques sont très différents, mais Macron et Poutine ont tous deux subi une sorte de déformation professionnelle $\mathbf{2 0}_{\lambda\rangle_{1}}$ a-t-il déclaré. Le manifeste accompagnant l'action parisienne renvoyait à un contexte historique très large, rappelant le rôle joué par la Banque de France dans l'échec de la Commune de Paris:

\section{La Banque de France a pris la place de la Bastille, les banquiers ont pris la place des monarques. La grande révolution française a fait de la France un symbole de liberté pour le monde. En 1917, grâce à ce symbole, la Russie s'est élancée vers la liberté. Mais, cent ans plus tard, la tyrannie règne de nouveau partout. La renaissance de la France révolution- naire déclenchera l'incendie mondial des révolutions. Dans ce feu, la Russie com- mencera sa libération $\mathbf{2 1}$.}

Au moment de la performance, en octobre 2017, le pathos de ce grand écart historique pouvait paraître, du moins à un public français initié, assez réducteur, un peu trop populiste ou naïvement grandiloquent. Le geste de Pavlenskiy s'inscrivait néanmoins dans la longue histoire (qu'on n'oserait qualifier de tradition) des iconoclasmes populaires dirigés contre les symboles communistes $\mathbf{2 2}$, lors des grands changements politiques dans les pays de l'ancien bloc socialiste et en ex-URSS (Gamboni 2015). Son action sur place de la Loubianka faisait écho à la destruction de la statue de Felix Dzerjynski (fondateur de la Tchéka, première institution du renseignement bolchevik) au cours des manifestations massives qui ont précédé la chute de I'URSS en août 1991. Sanctionnée par Mikhail Gorbatchev, la chute de la statue a permis de canaliser la violence des manifestants et d'éviter l'occupation du bâtiment du KGB, qui conservait alors tous les archives de la répression soviétique $\mathbf{2 3}$.

Dans La Destruction de l'art, Gamboni rappelle notamment qu'en 1989, lors de la destruction du mur de Berlin, les manifestants brandissaient des banderoles «1789-1989» (Gamboni 2015: 90). En 1989, à Berlin, la mise en avant de cette coïncidence historique réaffirmait la similitude des affects révolutionnaires éprouvés malgré des contextes et des époques aussi éloignés. Ce sont exactement ces mêmes sentiments - antiétatiques et antiautoritaires en Russie, anticapitalistes en France - que Pavlenskiy espérait retrouver ou provoquer à Paris.

Il a évoqué à ce sujet la destruction de la colonne Vendôme le 16 août 1871, supposément commanditée par Gustave Courbet. L'artiste en a en tout cas été reconnu coupable le 2 septembre 1871 lors d'un procès public et s'est vu condamné à six mois de prison, 500 francs d'amende et à la compensation des frais de reconstruction évalués à 323091 francs. Bien que ce rapprochement ne manque pas d'intérêt, Piotr Pavlenskyi semblait ignorer la place marginale de cet acte dans la reconnaissance posthume de Gustave Courbet. En France, Courbet n'est pas devenu «l'artiste politique» de la République. Au contraire, la dimension politique de son œuvre, malgré les tentatives récurrentes des spécialistes, demeure occultée par son grand style académique et surtout par l'effet «scandaleux» de L'Origine du monde, devenue l'œuvre centrale de l'artiste (Nochlin 1994; Clark 1969; Petitet 2014).

Ce type de gestes ne fait pas I'unanimité dans la France du xxle siècle, où «l'iconoclasme populaire» et l'usage du feu à des fins protestataires sont associés aux acteurs sociaux économiquement défavorisés et privés de légitimité politique: les habitants des banlieues, les jeunes délinquants et autres «vandales», récemment réunis sous la catégorie des «casseurs» dans la rhétorique médiatique et législative. Inversement, la destruction de monuments historiques a tendance à susciter de fortes «émotions patrimoniales» (Heinich 2012), comme on a pu le constater récemment avec l'incendie de Notre-Dame de Paris.

On peut tout de même accorder à Pavlenskiy I'intention de conquérir un nouvel auditoire par un geste visuellement spectaculaire, accessible à un très large public, et dépassant les codes esthétiques complexes des milieux de l'art contemporain. Mais cette intention démocratique allait ici se retourner contre lui. Une fois de plus, Pavlenskiy ne respectait pas les règles: ce geste le positionnait très loin de l'habitus culturel des amateurs d'art contemporain, dont l'approche élitiste favorise à l'inverse une pratique artistique «de recherche», complexe et très codifiée, où une médiation professionnelle s'avère souvent nécessaire pour en déchiffrer le message central.

Comparé à la Russie, le champ de l'art contemporain français bénéficie d'une plus grande autonomie socioprofessionnelle, ses frontières n'ayant pas été remises en cause depuis plus d'un demi-siècle (si l'on considère Mai 1968 comme un point de rupture); il est plus structuré, mais aussi plus hiérarchisé et autorégulé. Les gestes artistiques transgressifs, réservés aux galeries et aux centres d'art contemporain, ne portent que très rarement sur l'actualité politique et n'en sont eux-mêmes jamais une, contrairement, par exemple, à l'affaire des Pussy Riot en Russie. En France, les revendications politiques formulées par les artistes s'inspirent souvent des thématiques issues des mouvements politiques et sociaux mais n'en sont jamais à l'avant-garde. De manière générale, les artistes entretiennent avec le monde militant des liens de plus faible intensité en France qu'en Russie.

Le manque de compréhension du geste de Pavlenskiy proviendrait donc d'une double ignorance, ou d'une «double méprise» de l'audience: du point de vue des professionnels de l'art, cette forme d'iconoclasme-là n'a rien d'artistique, le temps de son «artification» (attribution de valeur et légitimation), comme cela s'est produit pour le graffiti et le street art, n'est pas encore venu. Et pour les mouvements
20. Entretien accordé à l'auteure le 25 décembre 2018.

21. Texte publié pour la première fois le 16 octobre 2017 sur le compte Twitter d'Inna Shevchenko, militante FEMEN, disponible sur: https://twitter. com/femeninna

(consulté le 7 janvier 2020).

22. En 2014, on a assisté à un retour de ce phénomène, avec la destruction collective de statues de Lénine en Ukraine, communément appelée Leninopad (à ce titre voir par exemple Vasylyvna 2015; Gobert 2018). Ce mouvement a pris par la suite une ampleur étatique, avec l'interdiction légale de tout symbole communiste dans l'Ukraine post-Maïdan.

23. À ce jour, le sort de cette statue n'est pas scellé et fait statue n'est pas scellé et fait
encore débat. Le conseil municipal de Moscou examine régulièrement des propositions de réhabilitation de celle-ci sur la place de la Loubianka, ou ailleurs en Rusie. Quant aux archives des services secrets soviétiques conservées à Moscou, elles n'ont jamais été rendues publiques. 
24. Article 322-6 du Code pénal français.

25. Rapport de police fourni par l'avocate de l'artiste.

26. Ordonnance du maintien en détention provisoire, numéro du parquet 1729100212, numéro d'instruction 2235/17/76, fournie par l'artiste.

27. Extraits des minutes du greffe du tribunal de grande instance de Paris, jugement du 10 janvier 2019, fourni parl'artiste.

28. Pour l'enregistrement de laquelle l'auteure a été l'interprète de Piotr Pavlenskiy, le 10 mai 2019.

29. Les bâtiments du tribunal de Paris et du Centre

Georges-Pompidou sont des Georges-Pompidou sont des
œuvres de l'architecte Renzo Piano, réalisées à trente-six an d'intervalle, respectivement en 2016 et en 1972. populaires, le recours à de tels gestes iconoclastes ne relève pas du domaine de l'esthétique: leurs acteurs, socialement éloignés du monde de l'art, ignorent tout simplement l'existence de l'«art politique», milieu avec lequel ils n'ont noué aucune alliance stratégique.

Cette observation contiendrait un premier élément de réponse à la question expérimentale de Pavlenskiy, formulée au début de cet article: «Son art [politique], sera-t-il traité différemment en France et en Russie? » Malheureusement pour lui, il semblerait que, en France, son geste ne soit ni politique ni artistique.

\section{L'ARTISTE RUSSE \\ FACE À LA JUSTICE FRANÇAISE}

Si la réception de son œuvre diffère entre la France et la Russie, sa traduction policière et juridique y est très semblable: immédiatement après les faits, Pavlenskiy a été arrêté et placé en garde à vue, puis mis en examen pour «destruction du bien d'autrui

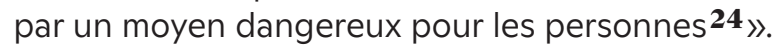

Tout comme à Saint-Pétersbourg ou à Moscou, à Paris, Piotr Pavlenskiy s'est comporté en «parfait suspect», n'opposant aucune résistance aux forces de l'ordre. Le rapport de police, établi par deux policiers en service ce matin-là, mentionne qu'«[il] a été interpellé sur place sans opposer aucune résistance, alors qu'il prenait la pose devant les flammes pour des photographes de presse tenant dans ses mains une boîte d'allumettes». Quant à sa compagne, «revêtue d'une perruque brune et de grandes lunettes de soleil [elle a été] interpellée alors qu'elle prenait la fuite à pied en essayant de se débarrasser

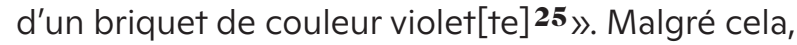
le juge d'instruction a estimé que l'artiste ne disposait pas de «garanties de représentation suffisantes» et que la détention était le seul moyen de maintenir «le prévenu à la disposition de la justice», en vue de «mettre fin à l'infraction ou de prévenir son renouvellement $26_{\Downarrow}$.

Dans la rhétorique juridique française, l'artiste de renommée internationale, «mondialement connu pour ses performances choquantes, politisées et hostiles au gouvernement russe actuel», est requalifié de façon peu glorieuse en migrant d'un pays de l'ex-bloc soviétique, «sans domicile fixe [...], inconnu de l'administration, [n'ayant] aucun emploi déclaré, [n'étant] titulaire d'aucun compte bancaire et ne [percevant] aucune aide sociale», qui «n'a pas cherché à sortir de la marginalité depuis son arrivée sur le territoire français» et «revendique de vivre sans travailler, sans rien payer et en squattant $\mathbf{2 7}_{\text {}}$.

Ainsi, Piotr Pavlenskiy a passé deux fois plus de temps en détention en France qu'en Russie, toutes performances réunies. Sur les onze mois de détention provisoire à la maison d'arrêt de la prison de Fleury-Mérogis, plus de deux mois ont été passés dans une cellule disciplinaire et six en cellule d'isolement, suite à des actes de désobéissance au règlement carcéral, que l'artiste jugeait, dans l'entretien qu'il nous a accordé en décembre 2018, «contraire à sa dignité». Lors de son incarcération, Pavlenskiy a mené deux grèves de la faim et de la soif, insistant pour que ses entretiens avec le juge soient rendus publics. En effet, en France, contrairement à la Russie, ces échanges se déroulent traditionnellement à huis clos. La requête de l'artiste, via plusieurs réclamations adressées à la chambre de l'instruction, a finalement été satisfaite. II exigeait également d'être transféré dans une cellule normale, où il pourrait voir d'autres prisonniers et insistait notamment sur son droit à la promenade quotidienne. II a obtenu gain de cause pour deux mois seulement, de juillet à août 2018, période que Pavlenskiy qualifie de «vacances d'été», où il a appris un peu le français grâce à ses échanges avec les autres détenus.

«À ma libération, aucun grand média français ne m'a interviewé pour parler de mon expérience en prison: cela ne semble intéresser personne», a affirmé Pavlenskiy lors d'une émission radiophonique enregistrée dans les locaux exigus de la radio associative Fréquence Paris Plurielle, dans le 19e arrondissement de Paris, au numéro 1 de la rue de la Solidarité. C'est donc au micro de l'émission hebdomadaire L'Envolée $\mathbf{2 8}$, lancée par d'anciens détenus et activistes du syndicat PRP (pour la protection et le respect des prisonniersères), que Pavlenskiy a pu parler de vive voix de son expérience pénitentiaire, non en tant qu'artiste dissident russe, mais en tant que Piotr Pavlenskiy, réfugié, ancien détenu de la prison de Fleury-Mérogis.

Cette étape carcérale semble indifférer la sphère médiatique française, illustrant presque à la lettre les propos de Michel Foucault sur l'occultation du processus même de la punition dans les sociétés modernes:

Désormais, le scandale et la lumière vont
se partager autrement; c'est la condam-
nation elle-même qui est censée marquer
le délinquant du signe négatif et uni-
voque: publicité donc des débats, et de
la sentence; quantà l'exécution, elle est
comme une honte supplémentaire que
la justice a honte d'imposerau condamné;
elle s'en tient donc à distance, tendant
toujours à la confier à d'autres, et sous
le sceau du secret.
(Foucault 1975: 25)

Il est vrai que le procès final de l'artiste, qui a eu lieu le 10 janvier 2019, avait un côté théâtral, mais ce spectacle était très différent de celui que la justice russe donnait à voir. Transposé dans le décor des nouveaux bâtiments du Palais de Justice érigés par Renzo Piano au nord de Paris, le procès avait entièrement perdu le sombre aspect kafkaïen que l'on ressentait dans les enfilades de couloirs de l'ancien Palais de l'île de la Cité. Par l'abondance de lumière naturelle, ses ascenseurs en acier et ses plafonds hauts, l'espace rappelait plutôt... un musée, dédié à l'art contemporain, une sorte de Centre Pompidou 29 de la justice. En filant la métaphore, on pourrait dire que le procès même de Pavlenskiy n'était pas le lieu d'un véritable débat, où l'affrontement des arguments 
déboucherait sur une décision juste, mais plutô† une sorte d'écrin, où la logique juridique française serait présentée et mise en scène, incarnée par la figure et le discours du juge.

Le juge, malgré le refus de Pavlenskiy de se lever, avait dans sa manière de s'adresser à lui quelque chose de bienveillant, voire d'enthousiaste. Dans son discours très érudit, il mettait en avant sa connaissance de l'art moderne et de la poésie, allant jusqu'à citer un dialogue imaginé par Jean Cocteau où le juge et l'artiste sont confrontés à Dieu. La leçon de cette fable moderniste, convoquée par le juge à des fins pédagogiques, énonçait qu'être artiste était une tâche dure et tragique, mais que selon Dieu, devoir juger était une tâche plus lourde encore. Le juge paraissait presque déçu de devoir passer la parole à Pavlenskiy, mais ce dernier lui a lancé: «Je parle la langue de l'art, vous parlez la langue du pouvoir, nous n'avons rien à nous dire, je ne répondrai plus à vos questions.»»

Ce procès final a reconnu l'artiste coupable: «Cette fois-ci, j'ai perdu», a reconnu Pavlenskiy, en sortant de la salle. «lls ont réussi à faire de mon art leur spectacle $\mathbf{3 0}^{\mathbf{} . »}$

\section{CONCLUSIONS}

La notoriété artistique de Pavlenskiy en Russie résultait de la triangulation circonstancielle de trois sphères: artistique, médiatique et juridique, dont les acteurs partagent un imaginaire politique commun, que l'artiste évoque de manière symbolique. Les conséquences juridiques de ses actes étaient vécues par son public comme une mise en procès de ses propres fantasmes révolutionnaires, ses procès rappelant la trajectoire d'un grand nombre d'individus, jugés pour leurs convictions politiques.

En Russie, I'art en tant que moyen d'expression symbolique semble inintelligible pour la justice, qui ne distingue pas la performance montée par l'artiste à des fins polémiques d'autres formes d'expression politique, qui pour des raisons historiques entrent dans le champ de vision de la justice pénale russe. Paradoxalement, c'est cette «cécité symbolique» de la justice qui est à l'origine de l'ouverture de l'art contemporain à des questions politiques en ex-URSS et de l'acceptation sociale des actes artistiques les plus transgressifs - tant qu'ils ont une lecture politique. C'est précisément cet effet de décloisonnement social, entraînant à la fois la complicité des acteurs juridiques et celle du large public engagé, qui permet à l'art de créer une rupture et un désarroi au sein de la sphère du droit, en subvertissant, bien que temporairement, sa logique, et en formulant un énoncé à caractère contre-hégémonique pour la tradition juridique russe.

À Paris, le geste de Pavlenskiy s'est heurté à une tout autre mécanique, dont les fondements culturels et structurels ont conditionné l'intraduisibilité de son geste sur le sol français. Dans la salle du tribunal, c'est le cas de Pavlenskiy qui, à l'inverse, a servi de toile de fond pour une mise en scène de l'autorité juridique, réaffirmant que le langage artistique, aussi subversif soit-il, ne peut s'affranchir de l'autorité primordiale du droit, susceptible de faire la différence entre l'art du crime sans recours et l'expertise des professionnels de l'art. Son histoire n'a donc produit aucun effet subversif au cœur de la tradition juridique française et a illustré de manière éloquente I'hégémonie du pouvoir actuel, où l'artiste obéit au juge, et le manifestant au policier.

\section{POST-SCRIPTUM: GILETS JAUNES}

Et pourtant, bien que qualifié de délit par décision du juge, et largement ignoré ou incompris par les milieux artistiques français, Éclairage avait quelque chose de prémonitoire dans le paysage parisien. Piotr Pavlenskiy réalisait sa performance une année exactement avant le début du mouvement des Gilets jaunes, apparu en France en octobre 2018. Une année plus tard, son action aurait pu être interprétée comme un gage de soutien au mouvement, même si les Gilets jaunes sont sociologiquement à l'opposé des milieux urbains amateurs d'art contemporain. Hormis la similitude de ses revendications antioligarchiques, le mouvement partageait avec Pavlenskiy les moyens d'action symbolique. Les Gilets jaunes ont même donné naissance à leur propre «Pavlenskiy»: le 29 décembre 2018, lors de l'acte VII des Gilets jaunes à Rouen, la porte de la Banque de France a été incendiée par un manifestant, dont le nom est resté inconnu $\mathbf{3 1}$.

Il est peu probable que ce manifestant ait été au fait des actions de Pavlenskiy. En revanche, I'artiste, ayant appris l'existence de son «épigone» français, a reconnu avoir ressenti «une satisfaction artistique

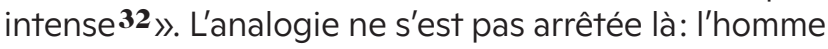
de 29 ans suspecté d'être le principal auteur de I'incendie a été interpellé un mois plus tard, pendant I'acte XI du mouvement, à Rouen. II a par la suite été déféré au parquet en vue de sa mise en examen pour «dégradations volontaires par incendie», dont le coût a été estimé à 30000 euros. En France, aucun commentateur (sauf, évidemment, Piotr Pavlenskiy en personne) n'a qualifié son geste d'œuvre artistique. Le procès n'a pas encore eu lieu, mais on peut déjà en deviner l'issue.
30. Communication personnelle de l'artiste.

31. «Incendie de la Banque de France, à Rouen : un suspect interpellé pendant l'acte XI des Gilets jaunes », 76Actu [en liǵne], disponible sur: https://actu.fr/normandie/ rouen_76540/incendiebanque-france-rouen-suspectinterpelle-pendant-lacte-xiinterpele-pendant-lacte-xi(consulté le 7 janvier 2020).

32. Entretien avec l'auteure du 25 décembre 2018 . 


\section{Études et essais}

\section{BIBLIOGRAPHIE}

\section{Barthes, Roland}

2015 Mythologies. Paris, Seuil.

Beumers, Birgit, Etkind, Alexander, Gurova, Olgá et Turoma, Sanna

2017 Cultural Forms of Protest in Russia. Londres, Routledge.

Clark, Timothy J.

1969 «A Bourgeois Dance

of Death: Max Buchon on

Courbet», The Burlington

Magazine 111 (793): 208-213.

\section{Citton, Yves}

2012 «Contre-fictions: trois modes de combat», Multitudes 48 $72-78$

\section{Ewick, Patricia}

et Silbey, Susan S.

$\mathbf{2 0 0 3}$ «Narrating Social Structure: Stories of Resistance to Legal Authority », American Journal of Sociology 108 (6) : 1328-1372.

1995 «Subversive Stories

and Hegemonic Tales: Toward a Sociology of Narrative», Law \& Society Review 29 (2) $197-226$.

Foucault, Michel

1975 Surveiller et punir: naissance de la prison. Paris, Gallimard.

\section{Fureix, Emmanuel (dir.)}

$\mathbf{2 0 1 4}$ Iconoclasme et révolutions: de 1789 à nos jours. Ceyzérieu, Champ Vallon.

Gabowitsch, Mischa

2016 Protest in Putin's Russia. Hoboken, John Wiley \& Sons.

Gamboni, Dario

2015 La Destruction de l'art: iconoclasme et vandalisme depuis la Révolution française.

Paris, Les presses du réel.

\section{Gobert, Sébastien}

2018 «En Ukraine, le passé toujours vivant», Études 5: 19-30.

\section{Goody, Jack}

2006 La Peur des représentations: l'ambivalence à l'égard des images, du théâtre, de la fiction, des reliques et de la sexualité, trad. de l'anglais par Pierre-Emmanuel Dauzat.

Paris, La Découverte.

\section{Heinich, Nathalie}

2012 «Les émotions patrimoniales: de l'affect à l'axiologie», Social Anthropology/Anthropologie sociale 20 (1): 19-33.

\section{Israël, Liora}

2009 L'Arme du droit. Paris, Presses de Sciences Po.

\section{Jonson, Lena}

2015 Art and protest in Putin's Russia. New York, Routledge.

\section{Nochlin, Linda}

1994 «The Depoliticisation of Gustave Courbet: Transformation and Rehabilitation under the Third Republic», in Michael Orwicz (dir.), Art Criticism and Its Institutions in Nineteenth-Century France.

Manchester, Manchester

University Press.

\section{Pavlenski, Piotr}

2016 Le Cas Pavlenski: la politique comme art, trad. du russe par Galia Ackerman. Paris, Louison éditions.

\section{Petitet, Cécile}

2014 «Courbet vandale et anti-vandale», Transversales 1 [en ligne], disponible sur: http:// tristan.u-bourgogne.fr/CGC/ publications/Transversales/ publications/Transversales/
Vandales_vandalismes/C_Petitet. html (consulté le 7 janvier 2020).

\section{Salmon, Christian}

$\mathbf{2 0 1 3}$ Storytelling: la machine à fabriquer des histoires et à formater les esprits. Paris, La Découverte.

\section{Silbey, Susan S.}

$\mathbf{2 0 0 5}$ «After Legal Consciousness», Annual Review of Law and Social Science 1 (1) : 323-368.

\section{Vasylyvna, Myrosh Mariya}

2015 «Human Geographical Aspects of Ideological Protests in Western Region of Ukraine », European Science Review 11-12: 208-209. 
165 